\title{
Star formation and AGN activity in a sample of local luminous infrared galaxies through multiwavelength characterization
}

\author{
Rubén Herrero-Illana, ${ }^{1,2 \star}$ Miguel Á. Pérez-Torres, ${ }^{1,3}$ Zara Randriamanakoto, ${ }^{4}$ \\ Antxon Alberdi, ${ }_{1}^{1}$ Andreas Efstathiou, ${ }^{5}$ Petri Väisänen, ${ }^{6,7}$ Erkki Kankare, ${ }^{8}$ \\ Erik Kool, ${ }^{9,10}$ Seppo Mattila, ${ }^{11,12}$ Rajin Ramphul ${ }^{4,6}$ and Stuart Ryder ${ }^{9}$ \\ ${ }^{1}$ Instituto de Astrofísica de Andalucía (IAA-CSIC), Glorieta de la Astronomía s/n, E-18008 Granada, Spain \\ ${ }^{2}$ European Southern Observatory (ESO), Alonso de Córdova 3107, Vitacura, Casilla 19001, Santiago de Chile, Chile \\ ${ }^{3}$ Visiting Scientist: Departamento de Física Teórica, Facultad de Ciencias, Universidad de Zaragoza, E-50009, Zaragoza, Spain \\ ${ }^{4}$ Astronomy Department, University of Cape Town, Private Bag X3, Rondebosch 7701, South Africa \\ ${ }^{5}$ School of Sciences, European University Cyprus, Diogenes Street, Engomi, 1516 Nicosia, Cyprus \\ ${ }^{6}$ South African Astronomical Observatory, PO Box 9, Observatory 7935, Cape Town, South Africa \\ ${ }^{7}$ Southern African Large Telescope, PO Box 9, Observatory 7935, Cape Town, South Africa \\ ${ }^{8}$ Astrophysics Research Centre, School of Mathematics and Physics, Queen's University Belfast, Belfast BT7 1NN, UK \\ ${ }^{9}$ Australian Astronomical Observatory, 105 Delhi Rd, North Ryde, NSW 2113, Australia \\ ${ }^{10}$ Department of Physics and Astronomy, Macquarie University, Sydney, NSW 2109, Australia \\ ${ }^{11}$ Tuorla Observatory, Department of Physics and Astronomy, University of Turku, Väisäläntie 20, FI-21500 Piikkiö, Finland \\ ${ }^{12}$ Finnish Centre for Astronomy with ESO (FINCA), University of Turku, Väisäläntie 20, FI-21500 Piikkiö, Finland
}

\begin{abstract}
Nuclear starbursts and active galactic nucleus (AGN) activity are the main heating processes in luminous infrared galaxies (LIRGs) and their relationship is fundamental to understand galaxy evolution. In this paper, we study the star formation and AGN activity of a sample of 11 local LIRGs imaged with subarcsecond angular resolution at radio $(8.4 \mathrm{GHz})$ and near-infrared $(2.2 \mu \mathrm{m})$ wavelengths. This allows us to characterize the central kpc of these galaxies with a spatial resolution of $\simeq 100 \mathrm{pc}$. In general, we find a good spatial correlation between the radio and the near-IR emission, although radio emission tends to be more concentrated in the nuclear regions. Additionally, we use an Markov Chain Monte Carlo code to model their multiwavelength spectral energy distribution (SED) using template libraries of starburst, AGN and spheroidal/cirrus models, determining the luminosity contribution of each component, and finding that all sources in our sample are starburst-dominated, except for NGC 6926 with an AGN contribution of $\simeq 64$ per cent. Our sources show high star formation rates (40-167 $\left.\mathrm{M}_{\odot} \mathrm{yr}^{-1}\right)$, supernova rates $\left(0.4-2.0 \mathrm{SN} \mathrm{yr}^{-1}\right)$ and similar starburst ages (13-29 Myr), except for the young starburst (9 Myr) in NGC 6926. A comparison of our derived starforming parameters with estimates obtained from different IR and radio tracers shows an overall consistency among the different star formation tracers. AGN tracers based on mid-IR, high-ionization line ratios also show an overall agreement with our SED model fit estimates for the AGN. Finally, we use our wide-band Very Large Array observations to determine pixel-by-pixel radio spectral indices for all galaxies in our sample, finding a typical median value $(\alpha \simeq-0.8$ ) for synchrotron-powered LIRGs.
\end{abstract}

Key words: galaxies: interactions - galaxies: nuclei - galaxies: starburst -infrared: galaxies radio continuum: galaxies.

\section{INTRODUCTION}

Luminous infrared galaxies (LIRGs) are defined as those galaxies with an infrared luminosity $L_{\mathrm{IR}}[8-1000 \mu \mathrm{m}]>10^{11} \mathrm{~L} \odot$. The majority of these sources, discovered in the early 1980s by the IRAS satellite, are actually galaxies undergoing a merging process. 
While most LIRGs are dominated by violent episodes of nuclear star formation (starbursts - SB; see e.g. Petric et al. 2011; Stierwalt et al. 2013), many of them also contain an active galactic nucleus (AGN). The existence and link between these two processes (e.g. Shao et al. 2010) make LIRGs ideal laboratories in which the AGN-SB connection is studied.

The parameters describing the star formation in a galaxy can be obtained by means of a number of indirect tracers and prescriptions based on different bands of the electromagnetic spectrum, including UV and far-infrared (FIR) continuum, as well as several recombination or forbidden lines (see review by Kennicutt 1998). Recently, near-IR observations have also proven useful to characterize the star formation properties of LIRGs through the study of super star clusters (SSCs; Portegies Zwart, McMillan \& Gieles 2010; Randriamanakoto et al. 2013a,b), which are young $(\simeq 10 \mathrm{Myr})$ massive-star clusters that preferentially form whenever there is strong ongoing SB activity. Other tracers are used to infer the AGN type, its luminosity and its relative contribution to the bolometric luminosity of galaxies, such as X-ray emission (Treister et al. 2009; Mullaney et al. 2011), mid-IR continuum (Asmus et al. 2014) or optical (e.g. Bassani et al. 1999; Heckman et al. 2005) and IR spectral lines (Genzel et al. 1998; Diamond-Stanic, Rieke \& Rigby 2009; Pereira-Santaella et al. 2010).

However, the large amount of dust present in LIRGs, whose reprocessing of ultraviolet photons from massive stars is responsible for the high IR luminosities in these systems, impose a limitation to optical and near-IR tracers due to dust obscuration (Mattila \& Meikle 2001). For this reason, observations at radio wavelengths, unaffected by dust extinction, are an alternative and powerful tool to trace $\mathrm{SB}$ and AGN processes in the innermost regions of these systems (Condon 1992; Parra et al. 2007; PérezTorres et al. 2009, 2010; Murphy et al. 2011; Romero-Cañizales, Pérez-Torres \& Alberdi 2012a).

There is a well-known tight linear correlation between the FIR and radio (1.4 GHz) luminosities (van der Kruit 1973; de Jong et al. 1985; Helou, Soifer \& Rowan-Robinson 1985; Condon 1992), with no evident dependence with redshift (Ivison et al. 2010; Pannella et al. 2015). To quantify this correlation, the so called $q$-factor (Helou et al. 1985) was defined as

$q=\log \left(\frac{\mathrm{FIR} / 3.72 \times 10^{12} \mathrm{~Hz}}{S_{1.49 \mathrm{GHz}}}\right)$,

where $S_{1.49 \mathrm{GHz}}$ is the flux density at $1.49 \mathrm{GHz}$ in units of $\mathrm{W} \mathrm{m} \mathrm{m}^{-2} \mathrm{~Hz}^{-1}$ and FIR is defined as

$\mathrm{FIR}=1.26 \times 10^{-14}\left(2.58 S_{60 \mu \mathrm{m}}+S_{100 \mu \mathrm{m}}\right)$,

with $S_{60 \mu \mathrm{m}}$ and $S_{100 \mu \mathrm{m}}$ being the IRAS fluxes in Jy at 60 and $100 \mu \mathrm{m}$, respectively. The mean value of the $q$-factor in the IRAS Bright Galaxy Sample (Condon \& Yin 1990) is $\langle q\rangle=2.34$ (Condon \& Broderick 1991; Yun, Reddy \& Condon 2001). While a radio excess $(q<2.34)$ is typically associated with a strong AGN contribution (e.g. Roy \& Norris 1997; Donley et al. 2005; Ivison et al. 2010; Del Moro et al. 2013) and an FIR excess $(q>2.34)$ is suggestive of intense star formation, this is not always the case (e.g. the obscured AGN in the most FIR-excess galaxy, NGC 1377; Costagliola et al. 2016). In general, the $q$-factor cannot be used as a standalone tool to separate AGN from SB galaxies (Morić et al. 2010; Padovani et al. 2011).

The underlying physics for the FIR-radio correlation is usually associated with a star formation origin. Dust reprocesses massivestar UV radiation into FIR photons, while the explosions of the same stars as supernovae ( $\mathrm{SNe}$ ) accelerate the cosmic ray electrons, responsible for the radio non-thermal synchrotron radiation (e.g. Voelk 1989; Lisenfeld, Voelk \& Xu 1996; Lacki \& Thompson 2010; Lacki, Thompson \& Quataert 2010). Thermal bremsstrahlung arising from $\mathrm{H}$ II regions (free-free emission) is also linked to this correlation (e.g. Murphy et al. 2011). Therefore, both thermal and non-thermal emission are correlated with the FIR and are good tracers of star formation. Correlations between radio emission and windows in the IR regime other than the FIR exist (e.g. 3.3 and $8.7 \mu \mathrm{m}$; as used for NGC 1614 in Alonso-Herrero et al. 2001; Väisänen et al. 2012; Herrero-Illana et al. 2014), but neither are as clear nor that well studied.

A problem of many of the SB indicators mentioned above is the frequent contamination of the tracers by the effects of an AGN. Notable examples are the contamination of the radio continuum by putative jets (Cleary et al. 2007) and the significant contribution to the FIR through the heating of the narrow-line region by the AGN (Tadhunter et al. 2007). A way to overcome this problem is to fit a multiwavelength spectral energy distribution (SED) combining templates of both SBs and AGN (e.g. Efstathiou, RowanRobinson \& Siebenmorgen 2000; Netzer et al. 2007; Mullaney et al. 2011; Calistro Rivera et al. 2016).

In this paper, we model the SED of a sample of 11 local LIRGs and compare our derived SB and AGN properties with other models. We also present radio $X$ band $(8.4 \mathrm{GHz})$ and near-IR $K_{S}$ band ( $2.2 \mu \mathrm{m}$; hereafter referred to as $K$ band) observations of this sample, comparing them and analysing possible near-IR/radio correlations. The paper is structured as follows: in Section 2 we present the sample, together with a concise individual description of the sources, and in Section 3, we present our observations and details on the data reduction. Through the discussion (Section 4), we describe our SED modelling in Section 4.1 and compare it with other tracers of star formation and AGN activity. We then compare our radio and near-IR observations (Section 4.2), analyse the special case of IRAS 16516-0948 (Section 4.3) and discuss the radio spectral index of our sources (Section 4.4). We summarize our results in Section 5.

Throughout this paper, we adopt a cosmology with $H_{0}=75 \mathrm{~km} \mathrm{~s}^{-1} \mathrm{Mpc}^{-1}, \Omega_{\Lambda}=0.7$ and $\Omega_{\mathrm{m}}=0.3$.

\section{THE SAMPLE}

The sources in our LIRG sample are taken from the IRAS Revised Bright Galaxy Sample (IRBGS; Sanders et al. 2003), and fulfil the following criteria: $D<110 \mathrm{Mpc}$, luminosity $\log \left(L_{\mathrm{IR}} / \mathrm{L} \odot\right)>11.20$ and declination $\delta>-35^{\circ}$. We chose those criteria so that our $8.4 \mathrm{GHz}$ Karl G. Jansky Very Large Array (VLA) observations in A-configuration (angular resolution of $\simeq 0.3$ arcsec) could image the central kpc region of all galaxies with spatial resolutions of $\simeq 70-150 \mathrm{pc}$, which would allow us to disentangle the compact radio emission from a putative AGN from the diffuse, extended radio emission linked to an $\mathrm{SB}$, as well as potentially allowing the detection of other compact sources, e.g. individual SNe or supernova remnants (SNR). Furthermore, we excluded warm LIRGs with IRAS colour $f_{25} / f_{60}>0.2$ to prevent contamination from obscured AGN activity (e.g. Farrah et al. 2007), except for Arp $299\left(f_{25} / f_{60}=0.22\right)$, which is borderline, but was included in the study since it is one of the most luminous local LIRGs. A total of 54 out of 629 sources in the IRBGS satisfy the conditions above, from which ours is a representative sample. Finally, we also excluded galaxies with no nearby reference star to guide the adaptive optics (AO) observations with the VLT or the Gemini telescopes.

In Table 1, we show our final sample, which consists of 11 LIRGs (although the two components of one of them, Arp 299, are treated 
Table 1. Galaxy sample properties.

\begin{tabular}{|c|c|c|c|c|c|c|c|c|}
\hline Name & $\begin{array}{c}\text { RA } \\
(\mathrm{J} 2000)\end{array}$ & $\begin{array}{l}\text { Dec. } \\
(\mathrm{J} 2000)\end{array}$ & $\begin{array}{c}D_{\mathrm{L}} \\
(\mathrm{Mpc})\end{array}$ & Merger stage ${ }^{b}$ & $\begin{array}{c}\log \left(L_{\mathrm{IR}}\right) \\
\left(\mathrm{L}_{\odot}\right)\end{array}$ & $\begin{array}{c}\log \left(L_{1.4 \mathrm{GHz}}\right) \\
\left(\mathrm{erg} \mathrm{s}^{-1} \mathrm{~Hz}^{-1}\right)\end{array}$ & $q$-factor ${ }^{c}$ & $\begin{array}{c}\text { X-ray } \\
\text { classification }^{d}\end{array}$ \\
\hline MCG+08-11-002 & 054043.7 & +494141 & 77.2 & $\mathrm{~d}$ & 11.41 & 29.56 & 2.61 & - \\
\hline $\operatorname{Arp} 299^{a}$ & 112829.8 & +583343 & 47.7 & c & 11.88 & 30.27 & 2.30 & AGN \\
\hline ESO 440-IG058 & 120651.9 & -315654 & 100.5 & $\mathrm{~b}$ & 11.36 & 29.80 & 2.32 & - \\
\hline IC 883 & 132035.3 & +340822 & 100.0 & $\mathrm{~d}$ & 11.67 & 30.10 & 2.34 & AGN \\
\hline CGCG 049-057 & 151313.1 & +071332 & 59.1 & $\mathrm{~N}$ & 11.27 & 29.35 & 2.74 & No AGN \\
\hline NGC 6240 & 165258.9 & +022403 & 103.9 & $\mathrm{~d}$ & 11.85 & 30.74 & 1.83 & AGN \\
\hline IRAS 16516-0948 & 165424.0 & -095321 & 96.9 & $\mathrm{~d}$ & 11.24 & 29.91 & 2.08 & - \\
\hline IRAS $17138-1017$ & 171635.8 & -102039 & 75.9 & d & 11.42 & 29.66 & 2.47 & - \\
\hline IRAS $17578-0400$ & 180031.9 & -040053 & 58.6 & $\mathrm{~b}$ & 11.35 & 29.52 & 2.64 & No AGN \\
\hline IRAS $18293-3413$ & 183241.1 & -341127 & 77.8 & $\mathrm{c}$ & 11.81 & 30.21 & 2.33 & No AGN \\
\hline NGC 6926 & 203306.1 & -020139 & 81.9 & $\mathrm{~d}$ & 11.26 & 29.98 & 1.97 & AGN \\
\hline
\end{tabular}

Notes. IR luminosities and luminosity distances were obtained from Sanders et al. (2003), while radio 1.4 GHz luminosities come from Condon et al. (1998).

${ }^{a}$ Including both NGC $3690 \mathrm{~W}$ and NGC 3690E.

${ }^{b}$ From the visual inspection of the Spitzer-IRAC $3.6 \mu \mathrm{m}$ images (Stierwalt et al. 2013). The code is as follows: (N) non-merger; (a) pre-merger; (b) early-stage merger; (c) mid-stage merger; (d) late-stage merger.

${ }^{c}$ See equation (1) for the definition of $q$-factor (Helou et al. 1985).

${ }^{d}$ When not stated, X-ray data are not available or inconclusive. See individual description of the galaxies for the corresponding references.

separately), all of them included in the GOALS sample (Armus et al. 2009), together with their IR luminosity and luminosity distance. We also include the merger stage as derived from IRAC $3.6 \mu \mathrm{m}$ morphology (Stierwalt et al. 2013), and the $q$-factor values (Helou et al. 1985) obtained using the IRAS fluxes from the IRBGS and the $1.4 \mathrm{GHz}$ fluxes from the NRAO VLA Sky Survey (NVSS; Condon et al. 1998). An optical image of each source in our sample is shown in Fig. 1. In Fig. 2, we show the correlation between $L_{\mathrm{IR}}$ and the radio luminosity at $1.4 \mathrm{GHz}$ for the sources in our sample (plotted with a star symbol) compared with the resulting sources of a cross-match between the IRBGS and the New VLA Sky Survey by Yun et al. (2001).

A short individual description of each source, some of which have been barely studied, is shown below.

\subsection{MCG +08-11-002}

This galaxy, also named IRAS $05368+4940$, is a barred spiral galaxy (type SBab) with a complex morphology, in a late stage of merging (Stierwalt et al. 2013; Davies et al. 2016). Its midIR extended emission is clearly silicate-dominated (Díaz-Santos et al. 2011). Davies et al. (2016) found evidence of a possible preceding SB episode, likely linked to the previous encounter of the galaxy nuclei.

\subsection{Arp 299}

Arp 299 is one of the most luminous LIRGs in the local Universe, and for that reason one of the most studied systems. It is in an early merger stage according to Keel \& Wu (1995) or in a mid-stage according to Stierwalt et al. (2013) and Larson et al. (2016). Arp 299 is formed by two galaxies and exhibits two clear radio nuclei (Gehrz, Sramek \& Weedman 1983), A and B, and two secondary components, $\mathrm{C}$ and $\mathrm{C}^{\prime}$. The remaining compact structure, $\mathrm{D}$, is believed to be a background quasar, unrelated to the system (Ulvestad 2009). Each radio source is identified in Fig. 3. Several SNe have been recently discovered in the inter nuclear region (Mattila et al. 2010; Ryder et al. 2010; Herrero-Illana et al. 2012b; Kankare et al. 2014; Romero-Cañizales et al. 2014) and in nucleus B (Ulvestad 2009; Romero-Cañizales et al. 2011, Pérez-Torres et al., in preparation).
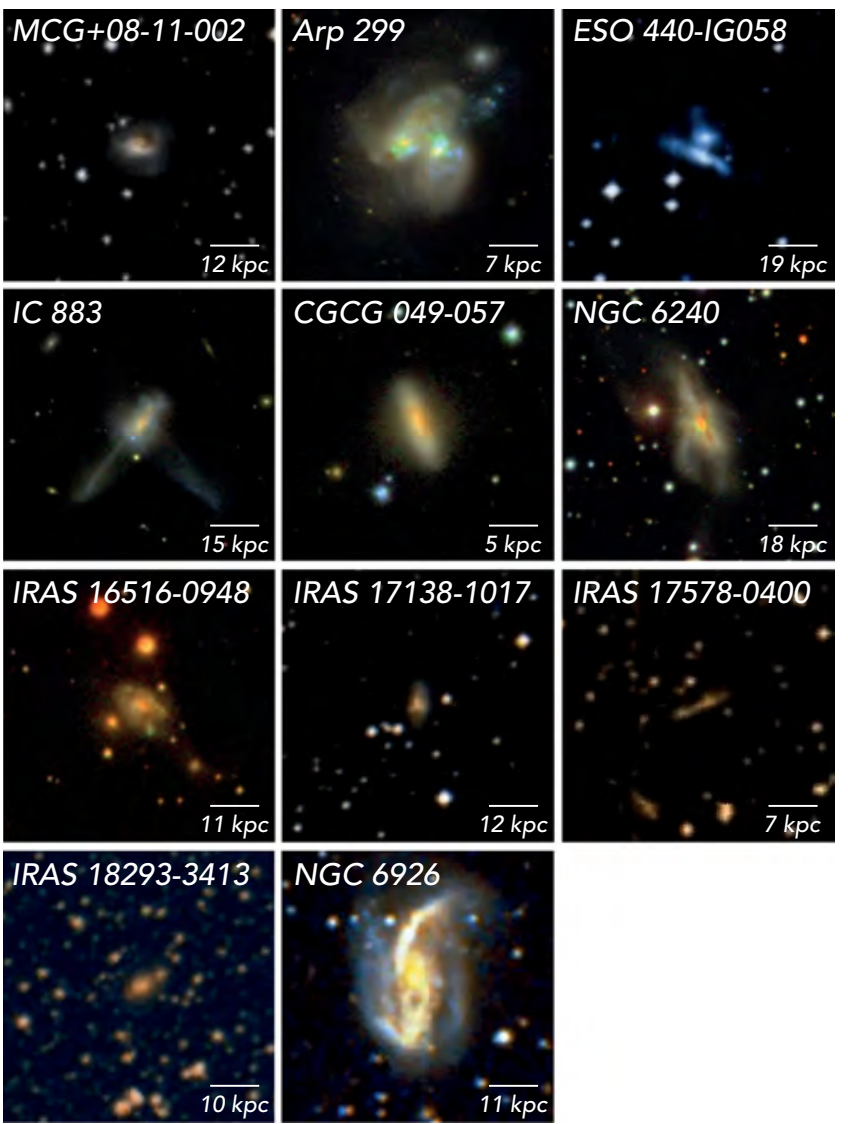

Figure 1. Optical colour composite images of the galaxies in our LIRG sample. Images are from the Sloan Digital Sky Survey (SDSS) DR9 (Ahn et al. 2012) when available, or from the STScI Digitized Sky Survey (DSS) otherwise.

However, it is the A nucleus that hosts a very rich SN factory (Neff, Ulvestad \& Teng 2004; Pérez-Torres et al. 2009; Ulvestad 2009; Bondi et al. 2012; Herrero-Illana, Pérez-Torres \& Alberdi 2012a), as well as a low-luminosity AGN, suggested from X-ray observations (Della Ceca et al. 2002; Zezas, Ward \& Murray 2003; Ballo et al. 2004), and found by means of very long baseline 


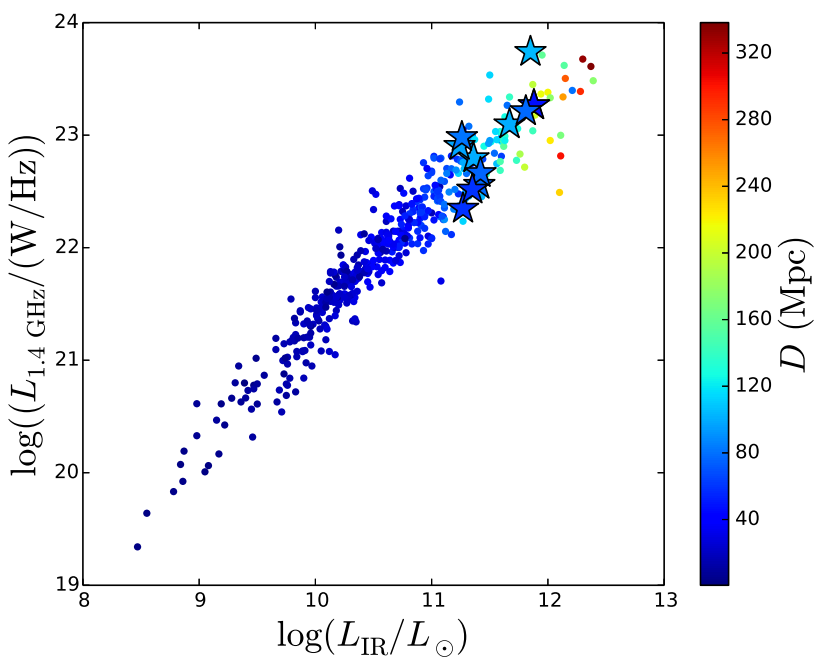

Figure 2. Radio $(1.4 \mathrm{GHz})$ versus infrared $(8-1000 \mu \mathrm{m})$ luminosity plotted for a sample of IRAS galaxies (dots) and our galaxy sample (stars), which falls into the LIRG regime. The most radio-powerful galaxy in our sample, NGC 6240, is also the one that deviates most from the correlation.

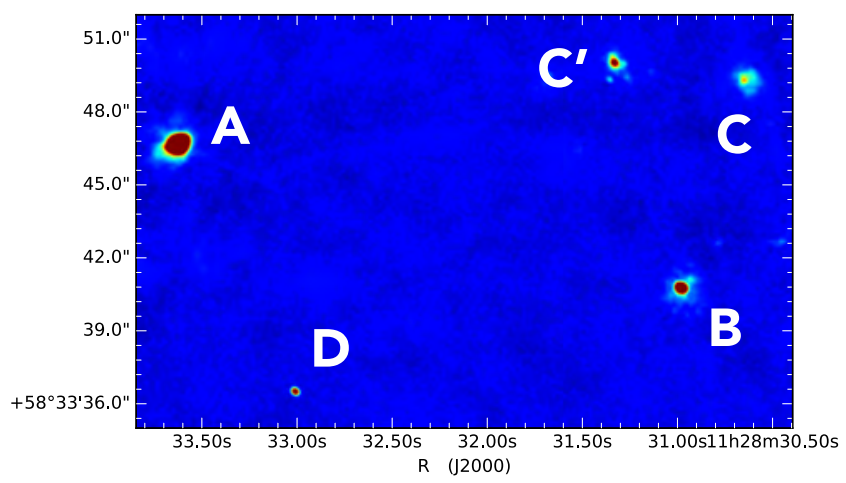

Figure 3. VLA $8.4 \mathrm{GHz}$ image showing the radio sources in the Arp 299 system. Nuclei A and B are the main components of NGC 3690 east and NGC 3690 west, respectively. Further details on the image can be found in Section 3.1

interferometry (VLBI) observations (Pérez-Torres et al. 2010). SED modelling for this system yielded a star formation rate (SFR) of 90 and $56 \mathrm{M}_{\odot} \mathrm{yr}^{-1}$ for the east and west components, respectively (Mattila et al. 2012).

While there are different nomenclatures to name the two galactic components of Arp 299, some have produced confusion in the literature (Corwin 2004). To avoid that, we use the unequivocal designations NGC 3690 east and NGC 3690 west for these components, which are treated individually throughout this paper.

\subsection{ESO 440-IG058}

This LIRG, also known as IRAS 12042-3140, consists of two merging galaxies separated by $\simeq 6 \mathrm{kpc}$. The northern component is very compact and has been classified as a LINER (Corbett et al. 2003). While the northern galaxy is dominated by star formation, the southern emission appears to be dominated by shocks ( $v=100-150 \mathrm{~km} \mathrm{~s}^{-1}$; Monreal-Ibero et al. 2010).

Based on the IR luminosity, Miluzio et al. (2013) estimated that ESO 440-IG058 has an SFR of $36 \mathrm{M}_{\odot} \mathrm{yr}^{-1}$ and an expected SN rate of $0.4 \mathrm{SN} \mathrm{yr}^{-1}$. Rodríguez-Zaurín et al. (2011) derived an age of the stellar population of $t \lesssim 6.5 \mathrm{Myr}$ for the currently star-forming stellar population.

\subsection{IC 883}

IC 883, also known as UGC 8387, is a late-merger LIRG at a distance of $100 \mathrm{Mpc}$, showing a peculiar morphology, with extended perpendicular tidal tails visible in the optical and near-IR (Smith et al. 1995; Scoville et al. 2000; Modica et al. 2012). This source was classified as an AGN/SB composite (Yuan, Kewley \& Sanders 2010), which has been confirmed by the direct detection of a number of radio components that are consistent with an AGN and with SNe/SNRs (Romero-Cañizales et al. 2012b) and with the detection, in the near-IR, of two SNe (Kankare et al. 2012) within the innermost nuclear region of the galaxy. Through SED model fitting, Romero-Cañizales et al. (2012b) estimated a core collapse supernova (CCSN) rate of $1.1 \mathrm{SN} \mathrm{yr}^{-1}$ and an SFR of $185 \mathrm{M}_{\odot} \mathrm{yr}^{-1}$. IC 883 also presents strong polycyclic aromatic hydrocarbon (PAH) emission, silicate absorption and a steep spectrum beyond $20 \mu \mathrm{m}$ (Vega et al. 2008). Recently, using radio VLBI and X-ray data, Romero-Cañizales et al. (2017) have reported unequivocal evidence of AGN activity, with the nucleus showing a core-jet structure and the jet having subluminal proper motion.

\subsection{CGCG 049-057}

Also known as IRAS $15107+0724$, this is the only LIRG in our sample classified as isolated. However, despite its apparent isolation (Larson et al. 2016), it has a complex and dusty nuclear morphology, so some form of past interaction cannot be ruled out. It hosts an $\mathrm{OH}$ megamaser (Bottinelli et al. 1986; Baan, Henkel \& Haschick 1987). It is optically classified as a pure SB, supported by Chandra X-ray observations (Lehmer et al. 2010). However, multiband radio observations show evidence of a buried AGN within the SB (Baan \& Klöckner 2006).

\subsection{NGC 6240}

This bright LIRG is a well-studied late-stage merger (Stierwalt et al. 2013). It hosts one of the few binary AGN detected so far using Chandra hard X-ray observations (Komossa et al. 2003), with a projected distance of $\simeq 1 \mathrm{kpc}$. This was later supported by the detection of two compact unresolved sources at radio wavelengths with inverted spectral indices $(\alpha=+1.0$ and +3.6 for the north and south components, respectively; see Gallimore \& Beswick 2004), finding also a third component with a spectral index consistent with a radio $\mathrm{SN}$. Close to the southern nucleus, a water-vapour megamaser was found (Nakai, Sato \& Yamauchi 2002; Sato et al. 2005).

\subsection{IRAS 16516-0948}

This unexplored LIRG was optically identified as a star-forming galaxy (Mauch \& Sadler 2007), and classified as a late merger from its infrared morphology (Stierwalt et al. 2013). IRAS 16516-0948 was part of the COLA project, for which high-resolution radio imaging did not detect any compact core (Corbett et al. 2002). Despite the scarce information available for this galaxy, we found it to be an interesting source, as discussed in Section 4.3. 
Table 2. Observation summary.

\begin{tabular}{|c|c|c|c|c|c|c|c|c|}
\hline \multirow[b]{2}{*}{ Name } & \multicolumn{4}{|c|}{ Radio $(8.4 \mathrm{GHz}$ or $3.6 \mathrm{~cm})$} & \multicolumn{4}{|c|}{ Near-IR $(2.2 \mu \mathrm{m})$} \\
\hline & $\begin{array}{l}\text { FWHM } \\
(\operatorname{arcsec})\end{array}$ & $\begin{array}{c}\mathrm{rms} \\
\left(\mu \mathrm{Jy} \mathrm{beam}^{-1}\right)\end{array}$ & $\begin{array}{c}\text { Peak } \\
\left(\text { mJy beam }^{-1}\right)\end{array}$ & $\begin{array}{l}\text { Int. flux } \\
\text { (mJy) }\end{array}$ & $\begin{array}{l}\text { Pixel size } \\
(\operatorname{arcsec})\end{array}$ & $\begin{array}{l}\mathrm{rms} \\
(\mu \mathrm{Jy})\end{array}$ & $\begin{array}{l}\text { Peak } \\
(\mathrm{mJy})\end{array}$ & $\begin{array}{c}\text { Int. flux } \\
\text { (mJy) }\end{array}$ \\
\hline MCG+08-11-002 & $0.26 \times 0.21$ & 8.64 & 0.73 & 16.68 & 0.022 & 0.06 & 0.006 & 21.27 \\
\hline NGC 3690W & $0.24 \times 0.20$ & 12.59 & 8.85 & 82.20 & 0.022 & 0.01 & 0.404 & 31.61 \\
\hline NGC 3690E & $0.24 \times 0.20$ & 16.91 & 24.85 & 22.13 & 0.022 & 0.03 & 0.017 & 93.90 \\
\hline ESO 440-IG058 & $0.60 \times 0.18$ & 8.12 & 1.07 & 6.39 & 0.02 & 0.007 & 0.006 & 21.07 \\
\hline IC 883 & $0.27 \times 0.21$ & 13.52 & 10.51 & 34.06 & 0.022 & 0.02 & 0.006 & 17.09 \\
\hline CGCG 049-057 & $0.24 \times 0.21$ & 23.77 & 19.73 & 26.94 & 0.022 & 0.02 & 0.002 & 17.89 \\
\hline NGC 6240 & $0.27 \times 0.20$ & 29.26 & 19.70 & 52.86 & 0.027 & 0.008 & 0.078 & 36.67 \\
\hline IRAS $16516-0948$ & $0.33 \times 0.20$ & 8.28 & 0.09 & 0.27 & 0.022 & 0.006 & 0.005 & 10.84 \\
\hline IRAS $17138-1017$ & $0.33 \times 0.20$ & 7.61 & 0.68 & 8.79 & 0.022 & 0.03 & 0.004 & 47.42 \\
\hline IRAS $17578-0400$ & $0.55 \times 0.21$ & 9.66 & 17.21 & 23.24 & 0.022 & 0.005 & 0.002 & 22.17 \\
\hline IRAS $18293-3413$ & $0.60 \times 0.17$ & 11.64 & 0.83 & 25.30 & 0.027 & 0.001 & 0.039 & 101.27 \\
\hline NGC 6926 & $0.25 \times 0.19$ & 7.26 & 3.99 & 6.00 & - & - & - & - \\
\hline
\end{tabular}

Notes. The angular resolution for the near-IR images is between 0.07 arcsec (diffraction-limited FWHM at $K$ band) and 0.10 arcsec. ESO $440-I G 058$ was observed with GeMS/GSAOI, NGC 6240 and IRAS 18293-3413 with NACO, and the remaining sources with ALTAIR/NIRI. Integrated fluxes are quoted above $5 \sigma$.

\subsection{IRAS 17138-1017}

This LIRG is a highly obscured SB galaxy (Depoy et al. 1988) in a late stage of interaction. An extremely extinguished SN $\left(A_{V}=15.7 \pm 0.8 \mathrm{mag}\right)$ was discovered in IRAS 17138-1017 using infrared $K$-band observations (SN2008cs; Kankare et al. 2008a). Two more SNe were also found: SN2002bw (Li 2002; Matheson et al. 2002) and SN2004iq (Kankare et al. 2008b).

\subsection{IRAS 17578-0400}

This is a galaxy pair in an early stage of merging (Stierwalt et al. 2013). Ultrahard X-ray (14-195 keV) observations with the Swift Burst Alert Telescope searching for AGN did not detect any compact emission in this source (Koss et al. 2013). There is abundant archival data in the X-ray, optical, IR and millimetre bands for this source (see e.g. Lu et al. 2014; Stierwalt et al. 2014; Rich, Kewley \& Dopita 2015), although no significant results for the purposes of this paper have been reported in the literature.

\subsection{IRAS 18293-3413}

This source was classified as an $\mathrm{H}_{\text {II }}$ galaxy based on its optical spectrum (Veilleux et al. 1995). It was detected in hard X-ray data (2-10 keV), obtained with $A S C A$, at a $5 \sigma$ level by Risaliti et al. (2000), finding no evidence of any AGN contribution to the X-ray spectrum. There are discrepancies on the merger stage determination. Stierwalt et al. (2013) classified it as a mid-stage merger from the visual inspection of Spitzer-IRAC $3.6 \mu \mathrm{m}$ images; on the other hand, Haan et al. (2011) classified it as a very early merger, with canonical discs and no tidal tails based on its Hubble Space Telescope (HST) morphology. However, Väisänen et al. (2008b), using high-resolution near-IR $K$-band AO imaging with VLT/NACO, showed the galaxy to have a very complex morphology, strongly suggesting a late-stage interaction. The system includes a rare unevolved elliptical companion as well. Using SED modelling, Mattila et al. (2007a) estimated a CCSN rate of $1 \mathrm{SN} \mathrm{yr}^{-1}$ for IRAS 18293-3413. SNe SN2004ip (Mattila et al. 2007b; Pérez-Torres et al. 2007) and AT2013if (Kool et al. 2017) were discovered within the nuclear regions of this galaxy.

\subsection{NGC 6926}

This relatively low luminosity LIRG is a spiral galaxy in a very early phase of interaction with the dwarf elliptical NGC 6929, located 4 arcmin to the east. Optically identified as a Seyfert 2 (Veilleux et al. 1995), NGC 6926 has a powerful water-vapour megamaser (Greenhill et al. 2003; Sato et al. 2005), typically found in heavily obscured AGN (e.g. Greenhill et al. 2003; Masini et al. 2016). Its X-ray hardness ratio (Terashima et al. 2015) is also suggestive of an AGN. This is the only source for which we lack near-IR observations.

\section{OBSERVATIONS AND DATA REDUCTION}

The observational data used in this study comes from (1) multiwavelength archival data obtained through the VizieR photometry tool (used for the SED model fits) and (2) from our own observations at both radio and near-IR wavelengths. Table 2 shows a summary of these observations. The images are shown in Fig. 4 and discussed in Section 4.2.

\subsection{Radio}

The radio data used in this paper were $X$-band $(8.4 \mathrm{GHz}, 3.6 \mathrm{~cm})$ observations in full polarization mode with a total bandwidth of $2048 \mathrm{MHz}$ (project 12B-105; PI: Pérez-Torres) taken between 2012 October 5 and December 26 using the new VLA capabilities. The total on-source time for each target was approximately $30 \mathrm{~min}$. For every source, we used 3C286 as the flux and bandpass calibrator, while we observed for each case a nearby bright point-like source for phase calibration purposes. We used the Common Astronomy Software Applications (CASA; McMullin et al. 2007) package for data reduction purposes, which consisted of standard amplitude and phase calibration. We imaged the sources using a Briggs weighting scheme with ROBUST $=0.5$ to obtain the best compromise between sensitivity and resolution. Table 2 shows the beam size, noise achieved and peak flux density for each image. To compare these images with our near-IR data, we converted the flux density units from $\mathrm{Jy}_{\text {beam }}{ }^{-1}$ to $\mathrm{Jy}_{\mathrm{px}}{ }^{-1}$.

Although our data were taken in a single band, the large bandwidth $(2 \mathrm{GHz})$ VLA $X$-band receivers allowed us to estimate the spectral index, $\alpha\left(S_{v} \propto v^{\alpha}\right)$, through this radio band. To this 

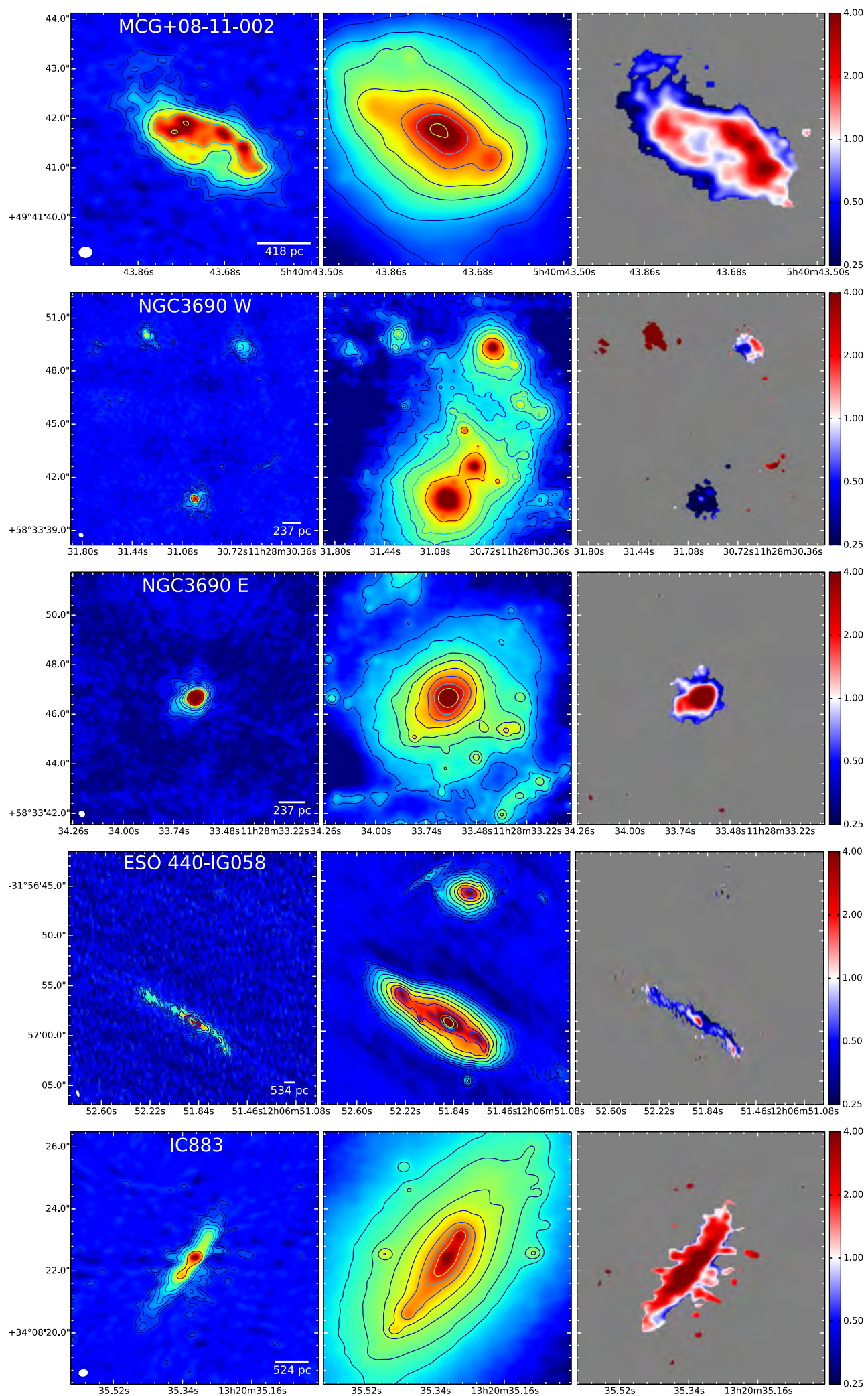

Figure 4. Radio and near-IR comparison of our sample. The image shows the $8.4 \mathrm{GHz}(3.6 \mathrm{~cm}$ ) radio maps (left), the $2.2 \mu \mathrm{m}$ IR maps (middle) and the ratio between radio and IR (right), where redder colours imply radio-dominated regions in contrast with bluer colours. Contours are drawn every $3(\sqrt{3})^{n} \times$ rms level, with $n=0,1,2,3, \ldots$ A direct comparison in a single plot between radio and near-IR is shown in Fig. A1. 

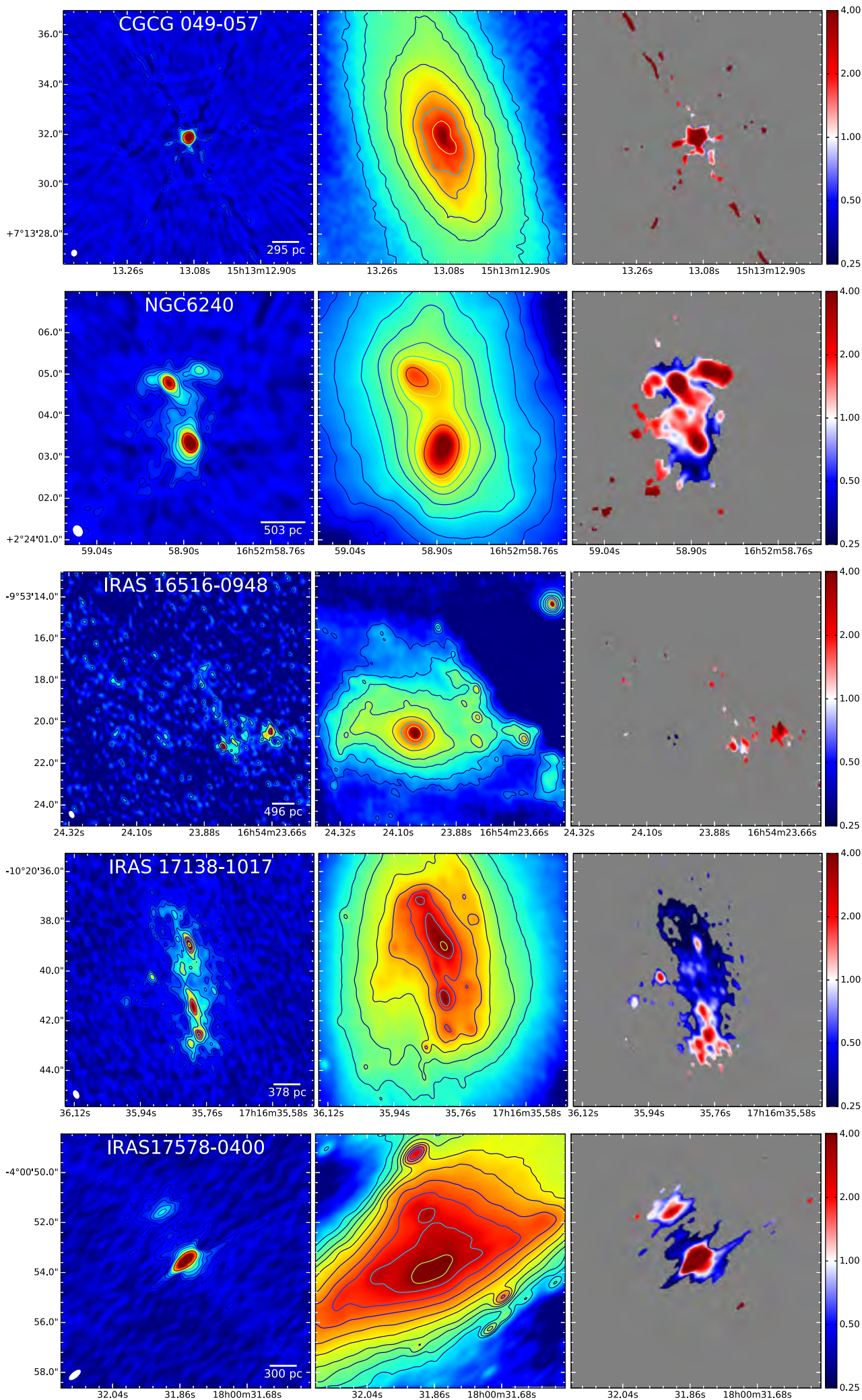

Figure 4 - continued

end, we imaged our sources using multiscale multifrequency synthesis (mode $=$ mfs within CASA; see Rau \& Cornwell 2011), which models the wide-band sky brightness as a linear combination of Gaussian-like functions whose amplitudes follow a Taylor- polynomial in frequency. The task performs a Taylor expansion to the second order (nterms $=2$ ) of the function:

$I_{v}^{\text {sky }}=I_{v_{0}}^{\text {sky }}\left(\frac{v}{v_{0}}\right)^{\alpha}$, 


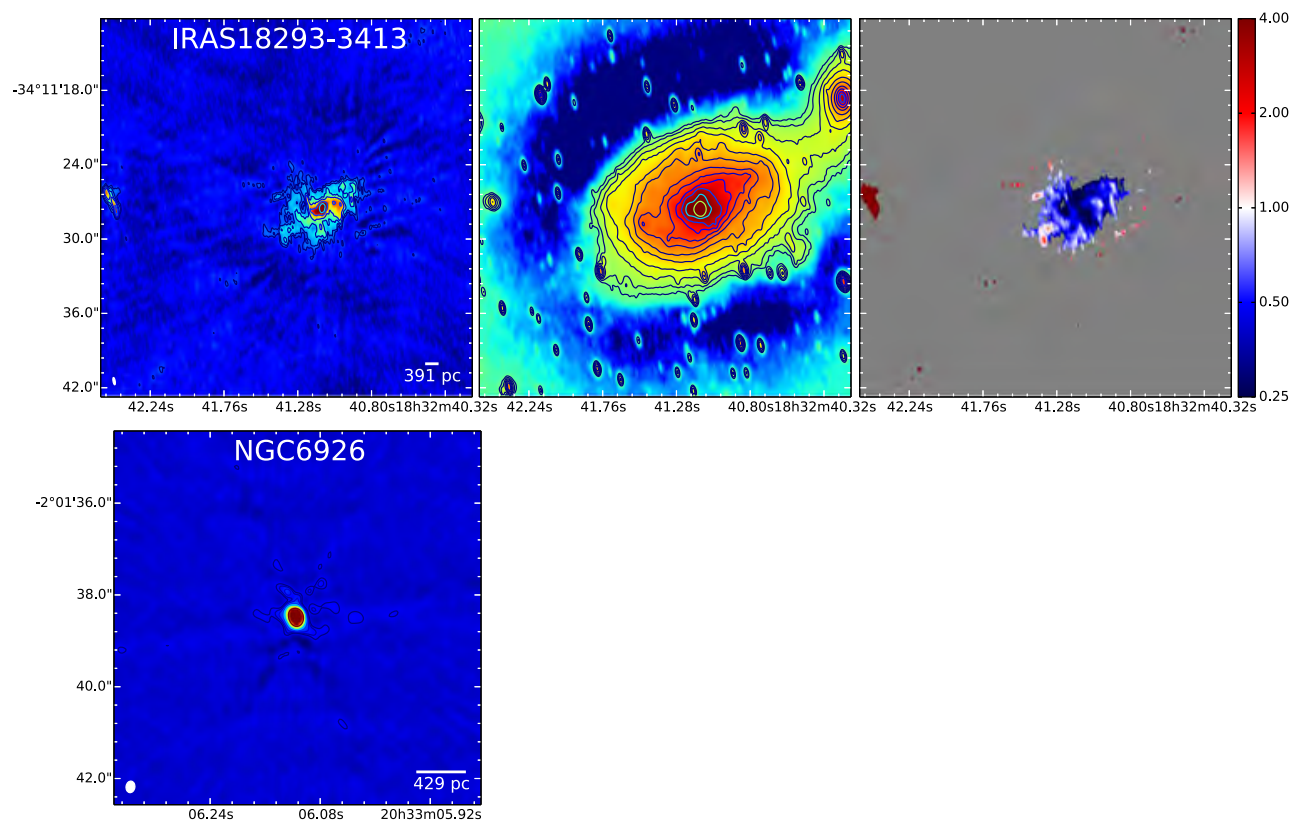

Figure 4 - continued

where $I^{\text {sky }}$ is the multiscale image, $\nu_{0}$ is the reference frequency (in our case $v_{0}=8.459 \mathrm{GHz}$ ) and $\alpha$ is the spectral index.

The two output images are the first two coefficients of the expansion, i.e.

$I_{0}=I_{v_{0}} \quad I_{1}=I_{v_{0}} \alpha$.

We then obtained the radio spectral index map of each source by simply using the ratio $I_{1} / I_{0}$. These maps are discussed in Section 4.4.

\subsection{Near-IR data}

We used ALTAIR/NIRI and GeMS/GSAOI on the Gemini North and South telescopes, respectively (PI: Ryder), and NACO on the VLT (PIs: Mattila) to obtain near-IR $K$-band $(2.2 \mu \mathrm{m})$ laser guide star $\mathrm{AO}$ images. These instruments have a pixel scale of $0.022 \operatorname{arcsec} \mathrm{px}^{-1}$ (ALTAIR/NIRI), $0.02 \operatorname{arcsec} \mathrm{px}^{-1}$ (GeMS/GSAOI) and $0.027 \operatorname{arcsec}^{\mathrm{px}^{-1}}$ (NACO, with camera S27). IRAS 18293-3413 and NGC 6240 were observed using NACO, ESO 440-IG058 using GeMS/GSAOI and the remaining targets were observed with ALTAIR/NIRI. Data from ALTAIR/NIRI come from a multi-epoch survey intended for the detection and study of nuclear CCSNe. The ALTAIR/NIRI images used in this paper are a combination of data from 2008 to 2012. NACO observations were performed on 2004 September 13 (IRAS 18293-3414) and 2011 May 31 (NGC 6240), and GeMS/GSAOI observations for ESO 440IG058 were performed on 2015 March 05 . The total integration time spans from $990 \mathrm{~s}$ (for NGC 6240) to $2192 \mathrm{~s}$ (for NGC 3690W). No near-IR data are available for NGC 6926. Spatial resolution was typically $\simeq 0.1$ arcsec in the AO corrected images.

We reduced the near-IR data using standard IRAF-based tasks, including flat-fielding and sky subtraction, and created the final images by average-combining individual frames from different epochs after having shifted them to a common reference. A detailed description of the reduction process of the ALTAIR/NIRI and NACO/VLT data is presented in Randriamanakoto et al. (2013a), while GeMS/GSAOI data are part of the SUNBIRD survey
(Kool et al. 2017). In multi-epoch data, we found no evidence of variability among the different individual epochs above $2 \sigma$.

\subsection{Astrometry calibration and image convolution}

Since the field of view (FOV) of the near-IR images was small (between 22 and 54 arcsec on a side), we first calibrated larger FOV archival data from NOT or HST/Advanced Camera for Surveys (ACS) using stars from Guide Star Catalogue-2 or 2MASS $K_{S^{-}}$ band catalogue. We then added the WCS information in the FITS header of our targets using these intermediate images (see Randriamanakoto et al. 2013a, for details). We estimate the astrometry calibration uncertainty to be $\simeq 0.15$ arcsec.

Given the significantly different resolution of the radio and nearIR images (see Table 2), we convolved the near-IR images with a Gaussian with the size of the radio beam for each case, and then rebinned the near-IR images to the pixel size used in our radio images (0.04 arcsec).

\section{RESULTS AND DISCUSSION}

\subsection{SED modelling}

There is a wide variety of methods, based on data at different wavelengths, to determine the SB and AGN properties of galaxies. These are not always consistent and often depend on the individual environment and star-forming history of each galaxy. A more reliable method is to take advantage of multiwavelength observations and fit the SED to combined templates of SB and AGN to derive the relevant parameters (AGN contribution, SB age, star formation rate and $\mathrm{SN}$ rate). In this section, we obtain these parameters and compare them with those obtained with traditional tracers.

We modelled the multiwavelength SED for the sources in our sample. To this end, we combined libraries of SB models (Efstathiou et al. 2000; Efstathiou \& Siebenmorgen 2009), AGN torus models (Efstathiou \& Rowan-Robinson 1995; Efstathiou et al. 2013) and models of the spheroidal/cirrus component (Efstathiou et al., in 


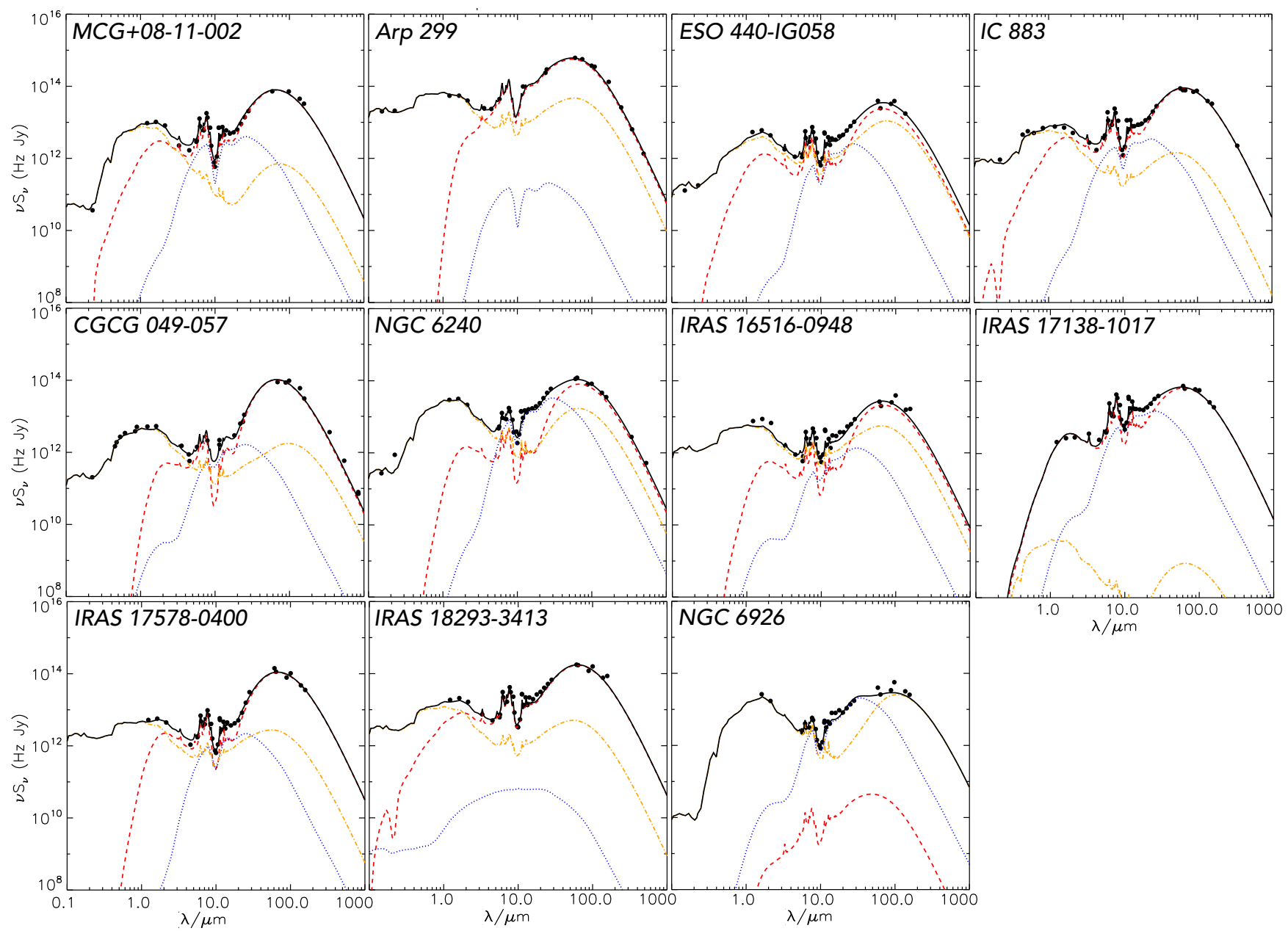

Figure 5. Best SED fitting model for all the sources in our sample. Black data points correspond to fitted data points (IRS spectral data are not plotted at their full resolution for the sake of clarity). The lines correspond to the SB contribution (dotted red), the AGN torus (dotted blue) and the spheroidal/cirrus component (dash-dotted orange). The solid black lines show the total ( $\mathrm{SB}+\mathrm{AGN}+$ spheroidal/cirrus) fit. See the main text for details.

preparation). The latter is based on cirrus models by Efstathiou \& Rowan-Robinson (2003) that rely on calculations of the radiative transfer problem in a medium where dust and stars are mixed in a spheroidal distribution. We used a Markov Chain Monte Carlo (MCMC) fitting code (SATMC; Johnson et al. 2013) to obtain realistic uncertainties on the fitted parameters. SATMC is based on Bayesian statistics, resulting in more reliable results and error determination than traditional least-square fitting. For details on the SATMC fitting process, we refer the reader to Johnson et al. (2013).

The SFR of the SB and its errors were derived self-consistently from the radiative transfer models that incorporate the stellar population synthesis model of Bruzual \& Charlot (1993, 2003). The libraries for a Salpeter initial mass function (IMF), solar metallicity and stars in the range $0.1-125 \mathrm{M}_{\odot}$ were used. The models of Efstathiou et al. predict the SED of an SB at different ages $t_{*}$ assuming that the SFR declines exponentially with an e-folding time $\tau$. From the SATMC fit, we know both the best-fitting values of $t_{*}$ and $\tau$ and their $1 \sigma$ uncertainties. Additionally, we know the fitted SB luminosity $L_{\mathrm{SB}}$ and the associated uncertainties. The combination of $L_{\mathrm{SB}}, t_{*}, \tau$ and the tables of Bruzual \& Charlot $(1993,2003)$ uniquely determined the total mass of stars formed in the SB episode for each combination. The stellar mass is divided by $50 \mathrm{Myr}$ to give the mean SFR that we provide.

We obtained the SED data points from public data through the VizieR photometry tool, which included NIR to FIR photometry
(2MASS, IRAC, WISE, AKARI, IRAS), as well as UV (GALEX), optical (SDSS) and (sub-)mm (SCUBA) data for some of the sources (see photometric data points in Fig. 5). All the photometric data we are using refer to the whole galaxy, either because the point spread function was big enough (FIR data) or because we used integrated fluxes. In addition, we included the Spitzer IRS spectra when available. For these, and to minimize aperture effects, we scaled up their fluxes in such a way that the edges of each spectrum match the flux of the adjacent photometric data points. The spectral resolution of the IRS data is reduced so that they are better matched to the resolution of the radiative transfer models. The IRS data included in the fitting with SATMC have a wavelength grid that is separated in steps of 0.05 in the log of rest wavelength. We additionally added more points around the $9.7 \mu \mathrm{m}$ silicate feature and the PAH features. There was no spectrum available for CGCG049-057, and we did not use IRS data for the fitting of Arp 299 either, since we are fitting both components together.

We show in Table 3 the best-fitting parameters, and in Fig. 5 we show the fitted SED for 10 sources. The CCSN rates are estimated by convolving the $\mathrm{SN}$ rate at a given time with the star formation history of the SB (see details in Mattila et al. 2012). For consistency, we modelled Arp 299 (NGC 3690E + NGC 3690W) as a unique galaxy but we note that a similar, yet not as complete model was published in Mattila et al. (2012) for the individual components. We note a discrepancy in the SB ages of NGC 3690E (45 Myr) 
Table 3. SED model fitting parameters.

\begin{tabular}{|c|c|c|c|c|c|c|c|}
\hline Name & $\begin{array}{c}L_{\mathrm{sph}} \\
\left(10^{10} \mathrm{~L}_{\odot}\right) \\
(2)\end{array}$ & $\begin{array}{c}L_{\mathrm{SB}} \\
\left(10^{10} \mathrm{~L}_{\odot}\right) \\
(3)\end{array}$ & $\begin{array}{c}L_{\mathrm{AGN}} \\
\left(10^{10} \mathrm{~L}_{\odot}\right) \\
(4)\end{array}$ & $\begin{array}{l}\text { AGN contribution } \\
\text { (per cent) } \\
(5)\end{array}$ & $\begin{array}{c}\mathrm{SFR}_{\text {mean }} \\
\left(\mathrm{M}_{\odot} \mathrm{yr}^{-1}\right) \\
(6)\end{array}$ & $\begin{array}{l}\mathrm{Age}_{\text {SB }} \\
(\mathrm{Myr}) \\
(7)\end{array}$ & $\begin{array}{c}v_{\mathrm{CCSN}} \\
\left(\mathrm{SN} \mathrm{yr}^{-1}\right)\end{array}$ \\
\hline $\mathrm{MCG}+08-11-002$ & $2.36_{-1.00}^{+1.26}$ & $21.52_{-0.58}^{+0.64}$ & $2.86_{-1.72}^{+5.99}$ & $10.7_{-6.1}^{+16.5}$ & $97.2_{-6.2}^{+2.3}$ & $29.1_{-0.8}^{+0.4}$ & $1.07_{-0.05}^{+0.02}$ \\
\hline Arp 299 & $15.31_{-2.83}^{+1.80}$ & $63.28_{-1.85}^{+0.96}$ & $0.06_{-0.05}^{+0.45}$ & $0.1_{-0.1}^{+0.6}$ & $86.1_{-18.4}^{+7.4}$ & $12.6_{-3.1}^{+2.6}$ & $1.45_{-0.22}^{+0.10}$ \\
\hline ESO 440-IG058 & $6.38_{-2.22}^{+1.39}$ & $9.65_{-1.25}^{+2.02}$ & $1.78_{-1.46}^{+1.89}$ & $10.0_{-8.1}^{+8.9}$ & $48.3_{-8.0}^{+11.1}$ & $27.9_{-3.4}^{+1.6}$ & $0.51_{-0.06}^{+0.11}$ \\
\hline IC 883 & $3.57_{-0.65}^{+7.93}$ & $37.02_{-1.63}^{+1.10}$ & $2.31_{-0.69}^{+1.21}$ & $5.4_{-1.5}^{+2.6}$ & $157.6_{-8.7}^{+8.6}$ & $28.6_{-1.8}^{+0.8}$ & $1.78_{-0.07}^{+0.07}$ \\
\hline CGCG 049-057 & $0.88_{-0.22}^{+0.57}$ & $11.21_{-0.35}^{+0.13}$ & $0.31_{-0.24}^{+0.30}$ & $2.5_{-1.9}^{+2.3}$ & $43.0_{-10.8}^{+4.5}$ & $24.8_{-5.0}^{+1.0}$ & $0.52_{-0.11}^{+0.03}$ \\
\hline NGC 6240 & $21.13_{-4.20}^{+7.70}$ & $33.93_{-8.03}^{+2.86}$ & $28.75_{-5.74}^{+11.97}$ & $34.3_{-4.5}^{+8.2}$ & $166.2_{-64.7}^{+22.6}$ & $27.6_{-3.7}^{+1.2}$ & $1.80_{-0.54}^{+0.18}$ \\
\hline IRAS $16516-0948$ & $5.55_{-0.89}^{+2.82}$ & $7.84_{-2.89}^{+0.78}$ & $1.35_{-1.35}^{+1.12}$ & $9.2_{-9.2}^{+7.0}$ & $40.3_{-18.8}^{+2.1}$ & $28.5_{-4.1}^{+0.3}$ & $0.42_{-0.17}^{+0.02}$ \\
\hline IRAS $17138-1017$ & $<0.05$ & $16.45_{-0.87}^{+0.87}$ & $4.54_{-1.27}^{+2.67}$ & $21.6_{-5.3}^{+9.7}$ & $58.8_{-3.2}^{+2.3}$ & $23.0_{-2.0}^{+1.3}$ & $0.75_{-0.04}^{+0.01}$ \\
\hline IRAS $17578-0400$ & $1.52_{-0.49}^{+0.38}$ & $14.84_{-0.24}^{+0.42}$ & $0.46_{-0.24}^{+0.42}$ & $2.7_{-1.4}^{+2.4}$ & $73.8_{-6.5}^{+5.4}$ & $27.7_{-1.5}^{+1.8}$ & $0.79_{-0.05}^{+0.04}$ \\
\hline IRAS $18293-3413$ & $5.44_{-2.18}^{+3.34}$ & $45.62_{-1.88}^{+0.86}$ & $0.02_{-0.01}^{+0.11}$ & $<0.2$ & $167.1_{-14.4}^{+11.8}$ & $27.5_{-0.5}^{+2.0}$ & $1.97_{-0.17}^{+0.07}$ \\
\hline NGC 6926 & $12.41_{-1.73}^{+2.42}$ & $0.01_{-0.01}^{+0.8}$ & $21.67_{-10.80}^{+6.41}$ & $63.6_{-19.5}^{+7.1}$ & $<0.5$ & $9.1_{-8.1}^{+5.2}$ & $<0.01$ \\
\hline
\end{tabular}

Notes. (1) Source name; (2) bolometric luminosity of the spheroid; (3) SB luminosity; (4) AGN luminosity (anisotropy corrected; see Efstathiou 2006); (5) percentage of AGN luminosity with respect to total luminosity; (6) SFR, averaged over the past 50 Myr; (7) SB age; (8) CCSN rate.

${ }^{a}$ NGC 3690 W and NGC 3690E were modelled by Mattila et al. (2012). The SB parameters from their SED modelling are quoted in the table.

and NGC 3690W (55 Myr) obtained by Mattila et al. (2012) and those derived here and also in the modelling by Alonso-Herrero et al. (2000) using the evolutionary synthesis models by Rieke et al. (1993) and Engelbracht et al. (1996), which yield significantly lower ages (6-8 and 4.5-7 Myr for NGC 3690E and NGC 3690W, respectively). However, we find the models not comparable since they are considering different IMF, different star formation history and, especially, different apertures: The whole system in this study, versus each component in Mattila et al. (2012) or the innermost $<5$ arcsec region in Alonso-Herrero et al. (2000). We also note that the higher SFRs quoted in Mattila et al. (2012) were averaged over the whole duration of the SB, while in this study we are averaging it over 50 Myr. None the less, the SN rate between both studies are compatible.

Our modelling allowed us to quantify the luminosity contribution of each component: the SB, the AGN and the spheroid, i.e. the underlying host galaxy stellar population minus the current SB. We find that the SED of all sources in our sample is SB-dominated except for NGC 6926 (AGN contribution of $\simeq 64$ per cent), with two additional sources having a significant AGN contribution ( 20 percent). Of the seven sources with an X-ray classification (see Table 1), four of them are catalogued as AGN: NGC 6926 and NGC 6240 show an important AGN contribution in our analysis, although Arp 299 and IC883 do not. However, we note that these two sources are known to host an AGN. We also derive SFR $\left(40-167 \mathrm{M}_{\odot} \mathrm{yr}^{-1}\right)$, SN rates $\left(0.3-2.0 \mathrm{SN} \mathrm{yr}^{-1}\right)$ and SB ages (23$55 \mathrm{Myr})$ that are consistent with their LIRG nature, with the exception of NGC 6926, a young SB (9 Myr) where SN events have not yet been triggered. The oldest SB of our sample corresponds to MCG+08-11-002 at $\simeq 29 \mathrm{Myr}$, while the youngest one is NGC 6926 at just $\simeq 9 \mathrm{Myr}$.

Three of our sources show a remarkably high CCSN rate (which depends on both SFR and age) above $1.5 \mathrm{SN} \mathrm{yr}^{-1}$ : IRAS 18293-3413 with $1.97 \mathrm{SN} \mathrm{yr}^{-1}$, NGC 6240 with $1.80 \mathrm{SN} \mathrm{yr}^{-1}$ and IC 883 with $1.78 \mathrm{SN} \mathrm{yr}^{-1}$, the latter consistent with previous studies $\left(v_{\mathrm{CCSN}}=1.1_{-0.6}^{+1.3}\right.$; Romero-Cañizales et al. 2012b). The extreme CCSN rate and SFR of IRAS 18293-3413, together with the controversy on its merger stage and its extremely steep spectrum ( $\alpha=-1.73 \pm 0.70$, see Section 4.4), turns it into an interesting case, likely hosting a very rich SB. This is supported by the de- tection of hundreds of SSCs in the field of IRAS 18293-3413 (Randriamanakoto et al. 2013a). We note however, the lower SN rate derived with a previous simpler model (1 $\mathrm{SN} \mathrm{yr}^{-1}$; Mattila et al. 2007a). By contrast, our fit for NGC 6926 is modelled by such a young SB (9 Myr) that no SN event has yet been observed from its current star formation episode. The ratio of the SB luminosity to $L_{\mathrm{IR}}$ from Table 1 (Sanders et al. 2003) is only 5 per cent for NGC 6926, in agreement with the above, while it ranges between 42 and 89 per cent for the rest of the sources.

In the following sections, we compare the results from our SED models described above with other proxies and indicators of both SFR and AGN.

\subsubsection{Comparison with SFR indicators}

Table 3 quotes the SFR obtained from the multiwavelength SED fit of our sources, averaged over the past 50 Myr. However, there is a large number of methods to indirectly estimate SFR in galaxies. From these prescriptions, only those that are not strongly affected by extinction (such as IR and radio tracers) are of real use in the dusty environments of LIRGs. In Fig. 6, we show a comparison between our SED modelling and three of these methods described below.

(i) The outcome of massive stars heating the interstellar dust is an intense infrared flux re-emitted by the dust through blackbody radiation. The following relation (Kennicutt 1998) is used to connect the infrared luminosity with the SFR in case of star formation $<100 \mathrm{Myr}$ of age, which is satisfied in the cases we present in this paper:

$\mathrm{SFR}=4.5 \times 10^{-44} L_{\mathrm{IR}}$

with SFR in $\mathrm{M}_{\odot} \mathrm{yr}^{-1}$ and $L_{\mathrm{IR}}$, measured between 8 and $1000 \mu \mathrm{m}$, in erg $\mathrm{s}^{-1}$, where we use the values quoted in Table 1 .

(ii) There is a tight correlation between the IR and radio luminosities (see Section 1). Thus, radio luminosity is expected to trace SFR in a similar way as $L_{\mathrm{IR}}$ does. A common prescription used to derive SFR from radio data (typically NVSS) is given by 


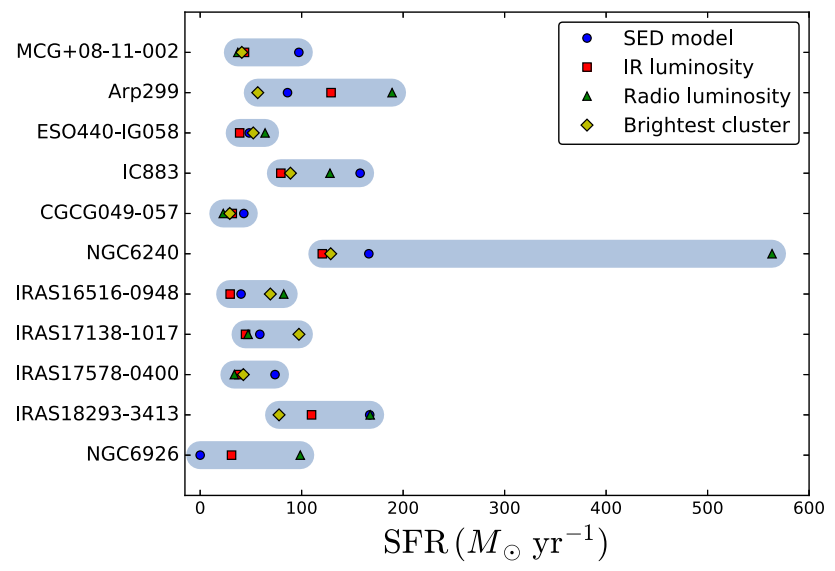

Figure 6. SFR SED models (blue circle) compared with three extinctionfree prescriptions, based on the IR luminosity (Kennicutt 1998, red squares), the radio luminosity (Murphy et al. 2011, green triangles) and the magnitude of the brightest SSC in the galaxy (Randriamanakoto et al. 2013b, yellow diamonds). The blue shaded area simply indicates the range between the lowest and the highest estimate for visual guidance. See the main text for details.

Murphy et al. (2011), and adapting it for a Salpeter IMF (as for the rest of the SFR indicators), we have

$\mathrm{SFR}=1.02 \times 10^{-28} L_{1.4 \mathrm{GHz}}$,

where SFR is given in $\mathrm{M}_{\odot} \mathrm{yr}^{-1}$ and $L_{1.4 \mathrm{GHz}}$ in $\mathrm{erg} \mathrm{s}^{-1} \mathrm{~Hz}^{-1}$, obtained from NVSS (Condon et al. 1998). The luminosity at this frequency is essentially due to non-thermal emission.

(iii) Randriamanakoto et al. (2013b) established a relation between the near-IR $K$-band magnitude of the brightest SSC in a galaxy and its global SFR. For the local Universe, this relation can be expressed as

$M_{k}^{\text {brightest }}=-2.56 \log (\mathrm{SFR})-13.39$.

The range between the highest and the lowest estimate of SFR (i.e. $\mathrm{SFR}_{\max }-\mathrm{SFR}_{\min }$ ) of the different tracers shown in Fig. 6 is approximately similar, with a median scatter of $78.1 \mathrm{M}_{\odot} \mathrm{yr}^{-1}$, with only one source, NGC 6240, deviating beyond the standard deviation, for which the SFR derivation through its radio luminosity is highly overestimated. The radio luminosity tracer is indeed known to overestimate the SFR in the presence of strong AGN activity (Del Moro et al. 2013; Bonzini et al. 2015), which is also supported by the large SFR derived from the radio luminosity in NGC 6926, with an AGN contribution of $\simeq 64$ per cent. In this sense, Fig. 7 shows the absolute and relative scatter of the SFR determination with respect to the relative AGN contribution. We suggest the existence of a weak trend, where more AGN-dominated sources present a larger scatter in the SFR determination from different proxies.

In the case of NGC 6240, Gallimore \& Beswick (2004) reported that no compact sources were detected with the Very Long Baseline Array (VLBA) at $8.4 \mathrm{GHz}$, with a $1 \sigma$ upper limit of $130 \mu \mathrm{Jy}_{\text {beam }}{ }^{-1}$, which might lead us to think that the AGN contamination is not a satisfactory explanation for the overestimated value of the radio SFR. However, the radio AGN emission has both compact and diffuse origin, and the large difference in angular resolution between the VLA and the VLBA can account for this apparent discrepancy. To test this, we fitted the $8.4 \mathrm{GHz}$ VLA emission from each nucleus to single Gaussian components, finding peak flux densities of 9.1 and $19.0 \mathrm{mJy} \mathrm{beam}^{-1}$ for the northern and southern components, respectively, out of a total emission of $53 \mathrm{mJy}$ (see Table 2). Given the

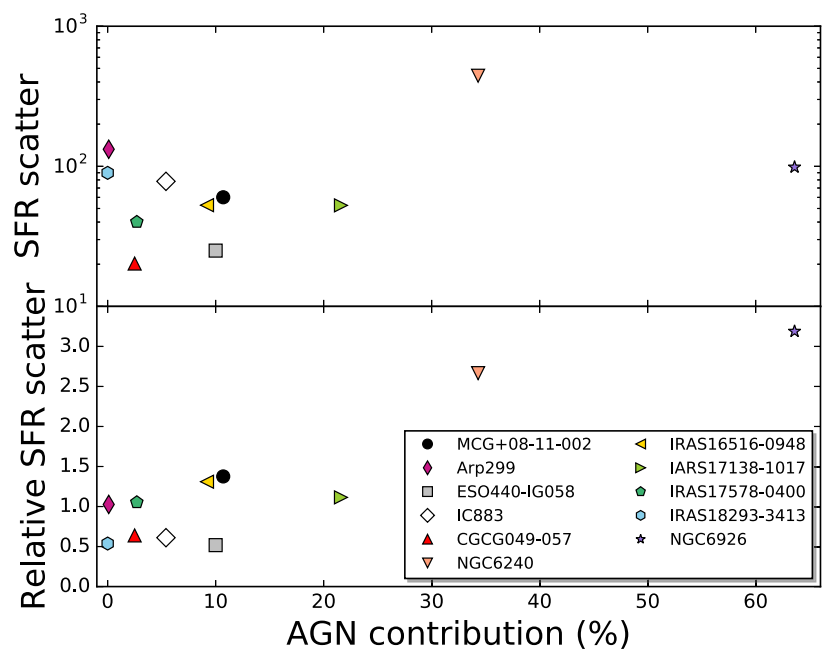

Figure 7. SFR scatter as a function of AGN contribution for each galaxy. Top panel shows the absolute scatter, given by $\mathrm{SFR}_{\max }-\mathrm{SFR}_{\min }$, while bottom panel shows the relative scatter, obtained through $\left(\mathrm{SFR}_{\max }\right.$ $\left.\mathrm{SFR}_{\min }\right) / \mathrm{SFR}_{\text {median }}$.

angular resolutions of the VLA $\left(0.27 \times 0.20 \operatorname{arcsec}^{2}\right)$ and the VLBA $\left(2.8 \times 1.1 \mathrm{mas}^{2}\right)$ observations, and assuming for simplicity that the total nuclear VLA emission $(28.1 \mathrm{mJy})$ is uniformly distributed, we would need an extreme VLBA sensitivity of $\sim 0.32 \mu \mathrm{Jy}_{\text {beam }}{ }^{-1}$ to detect the diffuse emission at $5 \sigma$. In summary, the radio AGN contamination is compatible with the available observations.

\subsubsection{Comparison with AGN indicators}

There are a number of AGN indicators in the mid-IR. In particular, we focused on the comparison between our SED modelling with three of these indicators: (i) the $6.2 \mu \mathrm{m}$ PAH equivalent width (EW); (ii) the $14.3 \mu \mathrm{m}[\mathrm{Ne}$ ] over $12.8 \mu \mathrm{m}$ [Ne II] line ratio and (iii) the $25.9 \mu \mathrm{m}\left[\mathrm{O}_{\mathrm{IV}}\right]$ over $12.8 \mu \mathrm{m}[\mathrm{Ne}$ II] line ratio.

Several authors have used the PAH features to estimate SFRs (e.g. Farrah et al. 2007; Shipley et al. 2016). However, in terms of EW, a lower PAH EW is found in AGN galaxies, either because PAH molecules are destroyed by the AGN radiation field (e.g. Voit 1992; Sales, Pastoriza \& Riffel 2010) or due to an increased AGN continuum contribution (Alonso-Herrero et al. 2014). Several PAH line EWs can be used to estimate the presence and contribution of AGNs. In particular, the feature at $6.2 \mu \mathrm{m}$ has the advantage of not being blended with other PAH features and is less affected by silicate absorption (Stierwalt et al. 2013). Pure SB galaxies have $6.2 \mu \mathrm{m}$ PAH EW values between 0.5 and $0.7 \mu \mathrm{m}$ (Brandl et al. 2006), while smaller values are usually indicative of an excess of hot dust due to the presence of an AGN (e.g. Genzel et al. 1998; Sturm et al. 2000; Wu et al. 2009).

High-ionization lines in the mid-IR (e.g. [Ne II], [Ne III], [Ne v], [O IV]) are also often used as tracers of AGN activity (e.g. Genzel et al. 1998; Armus et al. 2007; Pereira-Santaella et al. 2010; Dixon \& Joseph 2011), although some of these lines can be produced, with lower luminosities, in SN remnants (Oliva et al. 1999).

We obtained the $6.2 \mu \mathrm{m}$ PAH EW value for our sample from Stierwalt et al. (2013), while mid-IR line fluxes were taken from Inami et al. (2013). Table 4 shows these mid-IR indicators for our sample, where $1 \sigma$ upper limits are quoted for non-detections.

We have plotted these indicators together in Figs 8 and 9, as done before by other authors (Armus et al. 2007; Petric et al. 2011; 
Table 4. Mid-IR AGN properties.

\begin{tabular}{|c|c|c|c|c|c|c|}
\hline Name & $\begin{array}{c}6.2 \mu \mathrm{m} \text { PAH EW } \\
(\mu \mathrm{m})\end{array}$ & $\begin{array}{l}12.8 \mu \mathrm{m}[\mathrm{Ne} \text { II] } \\
\left(10^{-17} \mathrm{~W} \mathrm{~m}^{-2}\right)\end{array}$ & $\begin{array}{l}14.3 \mu \mathrm{m}[\mathrm{Ne} \mathrm{v}] \\
\left(10^{-17} \mathrm{~W} \mathrm{~m}^{-2}\right)\end{array}$ & $\begin{array}{l}25.9 \mu \mathrm{m}[\mathrm{O} \text { IV }] \\
\left(10^{-17} \mathrm{~W} \mathrm{~m}^{-2}\right)\end{array}$ & $\begin{array}{c}L_{\mathrm{AGN}}^{[\mathrm{Nev}]} \\
\left(10^{10} \mathrm{~L}_{\odot}\right)\end{array}$ & $\begin{array}{c}L_{\mathrm{AGN}} \\
\left(10^{10} \mathrm{~L}_{\odot}\right)\end{array}$ \\
\hline MCG+08-11-002 & $0.56 \pm 0.01$ & $67.3 \pm 0.6$ & $<2.5$ & $1.7 \pm 0.4$ & $<3.3$ & $2.36_{-1.00}^{+1.26}$ \\
\hline NGC 3690W & $0.12 \pm 0.02$ & $103.8 \pm 2.7$ & $<11.6$ & $28.9 \pm 3.8$ & $<5.7$ & - \\
\hline NGC 3690E & $0.38 \pm 0.03$ & $237.4 \pm 2.7$ & $<14.0$ & $16.1 \pm 14.3$ & $<6.6$ & - \\
\hline ESO 440-IG058 & $0.66 \pm 0.01$ & $48.9 \pm 0.4$ & $<2.9$ & $1.3 \pm 0.5$ & $<6.0$ & $1.78_{-1.46}^{+1.89}$ \\
\hline IC 883 & $0.62 \pm 0.01$ & $117.6 \pm 1.0$ & $1.6 \pm 0.1$ & $6.9 \pm 1.4$ & 9.9 & $2.31_{-0.69}^{+1.21}$ \\
\hline CGCG 049-057 & $0.51 \pm 0.04$ & $7.3 \pm 0.2$ & $<0.4$ & $<0.6$ & $<0.3$ & $0.31_{-0.24}^{+0.30}$ \\
\hline NGC 6240 & $0.35 \pm 0.01$ & $177.1 \pm 0.6$ & $3.4 \pm 0.2$ & $22.1 \pm 2.8$ & 21.6 & $28.75_{-5.74}^{+11.97}$ \\
\hline IRAS $16516-0948$ & $0.69 \pm 0.01$ & $27.4 \pm 0.3$ & $<0.8$ & $1.2 \pm 0.3$ & $<1.8$ & $1.35_{-1.35}^{+1.12}$ \\
\hline IRAS 17138-1017 & $0.68 \pm 0.01$ & $92.2 \pm 1.4$ & $<3.0$ & $<23.7$ & $<3.9$ & $4.54_{-1.27}^{+2.67}$ \\
\hline IRAS $17578-0400$ & $0.68 \pm 0.01$ & $46.1 \pm 0.7$ & $<3.2$ & $<8.7$ & $<2.4$ & $0.46_{-0.24}^{+0.42}$ \\
\hline IRAS $18293-3413$ & $0.63 \pm 0.01$ & $303.5 \pm 2.3$ & $<8.2$ & $7.4 \pm 1.0$ & $<10.2$ & $0.02_{-0.01}^{+0.11}$ \\
\hline NGC 6926 & $0.37 \pm 0.01$ & $7.3 \pm 0.1$ & $1.3 \pm 0.1$ & $4.6 \pm 0.2$ & 5.7 & $21.67_{-10.80}^{+6.41}$ \\
\hline
\end{tabular}

Notes. The PAH EWs at $6.2 \mu \mathrm{m}$ were obtained from Stierwalt et al. (2013), while the line fluxes come from Inami et al. (2013). We quote $3 \sigma$ upper limits for non-detections. The AGN bolometric luminosity obtained from the [Nev] flux $\left(L_{\mathrm{AGN}}^{[\mathrm{Nev}]}\right)$ is derived from equation (8). The last column is our SED-derived AGN luminosity (same as in Table 3), for comparison purposes.

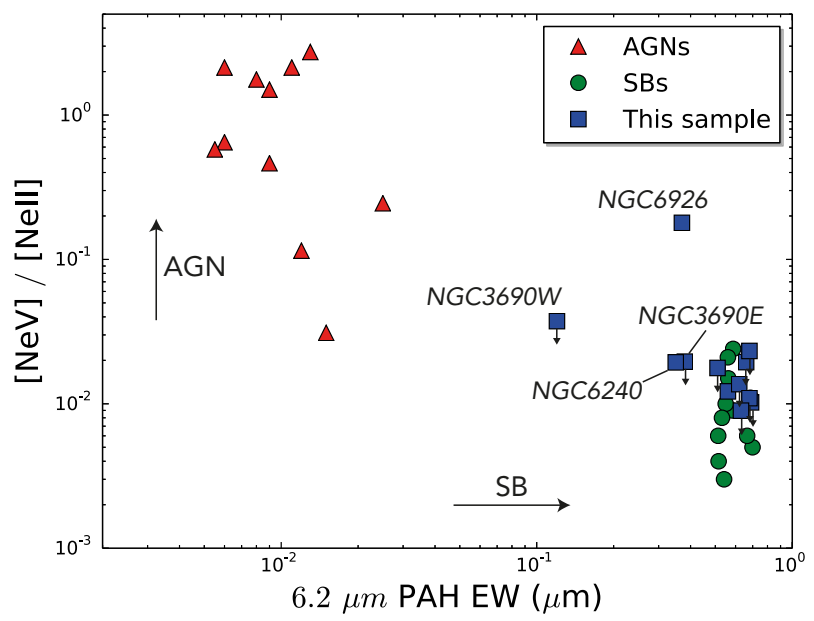

Figure 8. $[\mathrm{Ne} \mathrm{v}] /[\mathrm{Ne}$ II] against $6.2 \mu \mathrm{m}$ PAH EW. Our sample is plotted with blue squares. For comparison, we have also plotted a sample of AGN-dominated galaxies (red triangles; from Weedman et al. 2005), and a sample of SB-dominated sources (green circles; from Brandl et al. 2006).

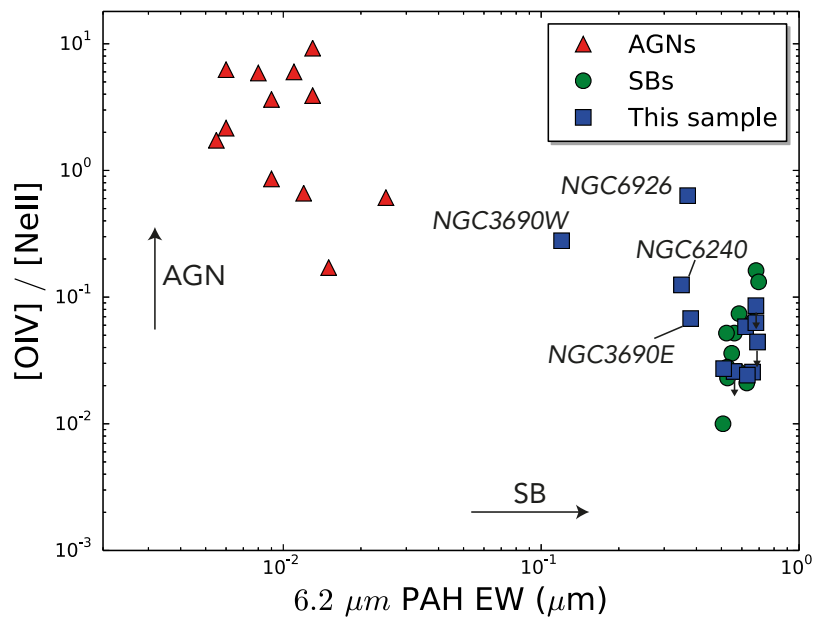

Figure 9. [O IV $] /[\mathrm{Ne}$ II $]$ against $6.2 \mu \mathrm{m}$ PAH EW. Colour codes and samples are the same as in Fig. 8. [O IV] is detected in a larger number of sources, despite having a lower ionization potential than [ $\mathrm{Ne} v]$.
Vardoulaki et al. 2015). In those figures, we have plotted the sources from our sample, together with two comparative samples: one for AGN-dominated systems (Weedman et al. 2005), and the other formed by SB-dominated galaxies (Brandl et al. 2006).

Two of the galaxies in our sample (NGC 3690W and NGC 6926) clearly diverge from the rest of the SB-dominated galaxies in the $[\mathrm{Ne} v] /[\mathrm{Ne}$ II] versus PAH EW diagram and two more (NGC 6240 and NGC 3690E) show a slight shift (see Fig. 8), which is more clear in the $[\mathrm{O} \mathrm{IV}] /[\mathrm{Ne} \mathrm{II}]$ versus PAH EW diagram shown in Fig. 9. The results shown by the mid-IR diagnostics are in a relatively good agreement with our model fits. In particular, NGC 6926 and NGC 6240 have a very important AGN contribution, although we would also expect IRAS $17138-1017$ to deviate from the SB-dominated loci in both diagnostic plots. With respect to NGC 3690W, Mattila et al. (2012) modelled its SED and found an important AGN contribution, compatible with the mid-IR diagnostics and previous observations (e.g. Ballo et al. 2004).

A more quantitative comparison can be made by means of the [Ne v] luminosity, $L_{[\mathrm{Ne} \text { v] }}$. Satyapal et al. (2007) proposed that $L_{[\mathrm{Ne} \text { v] }}$ is related to the AGN bolometric luminosity through:

$$
\log L_{\mathrm{AGN}}^{[\mathrm{Nev}]}=0.938 \log L_{[\mathrm{Nev}]}+6.317 \text {, }
$$

with luminosities measured in $\mathrm{erg} \mathrm{s}^{-1}$. Using the [Ne v] fluxes from Inami et al. (2013), we derived the luminosities quoted in Table 4. We find that the three sources with a [ $\mathrm{Nev}$ ] detection (IC 883, NGC 6240 and NGC 6926) do not follow the above correlation according to our SED-model derived AGN luminosities, albeit NGC 6240 is compatible within $2 \sigma$. For the remaining sources, there are only upper limits available that, although compatible with the correlation, do not give much information. The relation between $[\mathrm{Ne}$ v] and AGN luminosity arises from the high energetic environment ( $\simeq 97 \mathrm{eV}$ ) needed to produce $\mathrm{Ne}^{4+}$ (Abel \& Satyapal 2008), typically found around AGN. None the less, these energies can also be reached in young and extreme stellar populations (Kewley et al. 2001), contaminating this diagnostic tool.

\subsection{Radio and near-IR comparison}

There is a well-known tight correlation between the total IR luminosity, $L_{\mathrm{IR}}$, and the radio luminosity at $1.4 \mathrm{GHz}$, spanning five 
orders of magnitude, as shown in Fig. 2. In particular, from the sources in our sample, NGC 6240 is the galaxy with the strongest radio emission and the one that diverges more significantly from the trend. It is also the only source from our sample known to host a dual AGN, in agreement with the overestimated value in the SFR derived from the radio luminosity in Section 4.1.1.

The near-IR band, although presenting an overall correlation with radio emission (except for some remarkable cases discussed in this section), traces different processes. Red supergiants, used as indicators of young stellar populations $(\simeq 10 \mathrm{Myr})$, radiate most of their energy in the near-IR (Oliva et al. 1995). Near-IR also includes an important contribution from thermally pulsating asymptotic giant branch stars, with young to intermediate populations, up to 2 Gyr (Maraston 1998). This range of the spectrum can be used to effectively trace star formation in galaxies where the relatively high extinction hinders their study in the optical (e.g. Dametto et al. 2014). This makes near-IR a good spectral window in which (super) star clusters (e.g. Díaz-Santos et al. 2007; Randriamanakoto et al. 2013a) are studied. On the other hand, the radio emission at $3.6 \mathrm{~cm}$ is dominated by two mechanisms: radio thermal emission (tracing current star formation) and non-thermal synchrotron emission (tracing emission from $\mathrm{SNe}$ and $\mathrm{SN}$ remnants, i.e. older populations). This explains the general correlation between radio and near-IR, but at the same time, makes the interpretation of the ratio images problematic. In this sense, we find that it is not possible to use the ratios as age maps, unless we are able to, at least, disentangle the thermal and non-thermal radio emission (Tabatabaei et al. 2007; Basu \& Roy 2013; Herrero-Illana et al. 2014). Furthermore, although near-IR can efficiently penetrate through dust, very dust obscured regions can hinder even near-IR emission, adding an uncertainty to the age interpretation.

Fig. 4 shows both our radio $3.6 \mathrm{~cm}$ and near-IR $2.2 \mu \mathrm{m}$ subarcsec resolution images, as well as the flux density ratio between them (reddish for radio-dominated regions; bluish for near-IR-dominated ones; white implies a flux density ratio of unity). Any pixel that is not above three times the corresponding rms at both $3.6 \mathrm{~cm}$ and $2.2 \mu \mathrm{m}$ is masked out in the ratio maps. For a direct comparison between the radio and the original non-smoothed near-IR images, we refer the reader to Fig. A1. We find radio emission to be generally more concentrated in compact nuclear regions. Still, there is not a common trend in all the sources. Indeed, three LIRGs in our sample are completely radio-dominated in their common emission region: IC 883, CGCG 049-057 and IRAS 16516-0948, although near-IR extends further than the radio emission in the latter source.

In the remaining sources, radio and near-IR emission dominate in different regions. We remark, however, the radio silence of bright near-IR knots in three sources: ESO 440-IG058, IRAS 18293-3413 and especially IRAS 16516-0948, which will be discussed in Section 4.3. The northern source detected in IRAS 17578-0400 is not in this list, as it is a field star, which does not look like a compact Gaussian emission due to the image convolution with the radio beam. By contrast, IRAS 18293-3413 has an important compact radio emission $\simeq 18$ arcsec to the east of the main component that does not show a near-IR counterpart.

A particularly interesting case is NGC $3690 \mathrm{~W}$ (the western component of Arp 299). Its different knots show completely different behaviours regarding their radio and near-IR correlation. While the near-IR dominates the emission of the southern nucleus (designated B in the literature; Gehrz et al. 1983), the north-west component (designated $\mathrm{C}$ ) has a comparable emission, and the northeast component $\left(\mathrm{C}^{\prime}\right)$ is very faint at $2.2 \mu \mathrm{m}$. This near-IR faintness was discussed by Alonso-Herrero et al. (2000), whose SB models predict a persistence of the emission at $2 \mu \mathrm{m}$ after their assumed Gaussian burst with a full width at half-maximum (FWHM) of 5 Myr. They concluded then that the star formation episode must have been shorter in $C^{\prime}$. Indeed, Leitherer \& Heckman (1995) models nicely fit an instantaneous burst, which yields a mass of the SB episode of $3 \times 10^{7} \mathrm{M}_{\odot}$, with an age of $4 \mathrm{Myr}$, supporting this idea.

Another interesting case is NGC 6240. The nature of the two main compact structures is well determined as a dual AGN (Komossa et al. 2003; Gallimore \& Beswick 2004; Risaliti et al. 2006). The nature of the fainter north-west radio component, however, is not so clear. This component was first reported by Colbert, Wilson \& Bland-Hawthorn (1994) at $3.6 \mathrm{~cm}$, estimating its $L_{\mathrm{IR}}$ to be $\simeq 2 \times 10^{10} \mathrm{~L} \odot$. They suggested that, considering its steep spectral index $(\alpha=-1.1)$, it could be a clump of ejected electrons (a superwind; Heckman, Lehnert \& Armus 1993), possibly ejected from a powerful SB in the nearby compact bright region. Beswick et al. (2001) found a consistent $1.4 \mathrm{GHz}$ structure using Multi-Element Radio-Linked Interferometer (FWHM: 0.31 arcsec $\times 0.15$ arcsec), but did not detect the source at $5.0 \mathrm{GHz}$ with the same instrument (FWHM: 0.10 arcsec $\times 0.06$ arcsec), due to the steep spectral index and the limited sensitivity at $5.0 \mathrm{GHz}$ to detect the diffuse emission. The peak reported by Colbert et al. (1994) for this relatively faint peak is $1.10 \mathrm{mJy} \mathrm{beam}^{-1}$, in agreement with our deeper VLA image $\left(1.06 \pm 0.23 \mathrm{mJy}^{-1}\right.$ beam $^{-1}$; see Fig. 4).

Finally, we did not find any correlation between the radio and near-IR total luminosities, nor between the radio to near-IR ratio maps and the position of the known SSCs in these galaxies (Randriamanakoto et al. 2013a).

\subsection{An off-nuclear star-forming region in IRAS 16516-0948}

Fig. 4 shows an unexpected lack of correlation between the studied bands for IRAS 16516-0948. Fig. 10 shows our near-IR and radio data contours overplotted on archival Spitzer images of IRAS 16516-0948. It is clear from the figure that at increasing wavelength the infrared peak is shifting its position towards the west. The surroundings of this galaxy present enough compact sources to ensure unequivocal relative astrometry. The shift in the peak of the $8.0 \mu \mathrm{m}$ with respect to the $3.6 \mu \mathrm{m}$ peak is $\simeq 5 \mathrm{arcsec}$. The radio contours correlate well with the peak at $8.0 \mu \mathrm{m}$.

The case of IRAS 16516-0948 is rare, but not unique among (U)LIRGs: a clear example is the merger II Zw 096 $\left[\log \left(L_{\mathrm{IR}} / \mathrm{L}_{\odot}\right)=11.94\right]$, where 80 percent of its total IR luminosity comes from a compact optical-invisible source $\simeq 5$ arcsec away from the optical peak (see fig. 2 in Inami et al. 2010), and can have an SFR density up to $780 \mathrm{M}_{\odot} \mathrm{yr}^{-1} \mathrm{kpc}^{-2}$. As in IRAS 16516-0948, radio emission from II Zw 096 correlates well with the MIPS peak, suggesting that, in the region with the strongest mid-IR and radio emission, intense star formation is occurring. Another interesting case is IRAS 19115-2124, or The Bird, the SB of which is dominated by an off-nucleus minor component of the interaction (Väisänen et al. 2008a, Väisänen et al. 2017).

Haan et al. (2011) suggested an explanation for this phenomenon: these galaxies are possibly not yet relaxed with off-nuclear SB and/or strong shocks, with the off-nuclear emission being associated with spiral arms, a possible secondary nucleus or the region between the merging nuclei. The sample by Haan et al. (2011) contains 73 LIRGs from the GOALS sample, and finds off-nuclear mid-IR emission in six of them $(\simeq 9.5$ per cent). A single case in our sample of 11 LIRGs agrees with this proportion. 


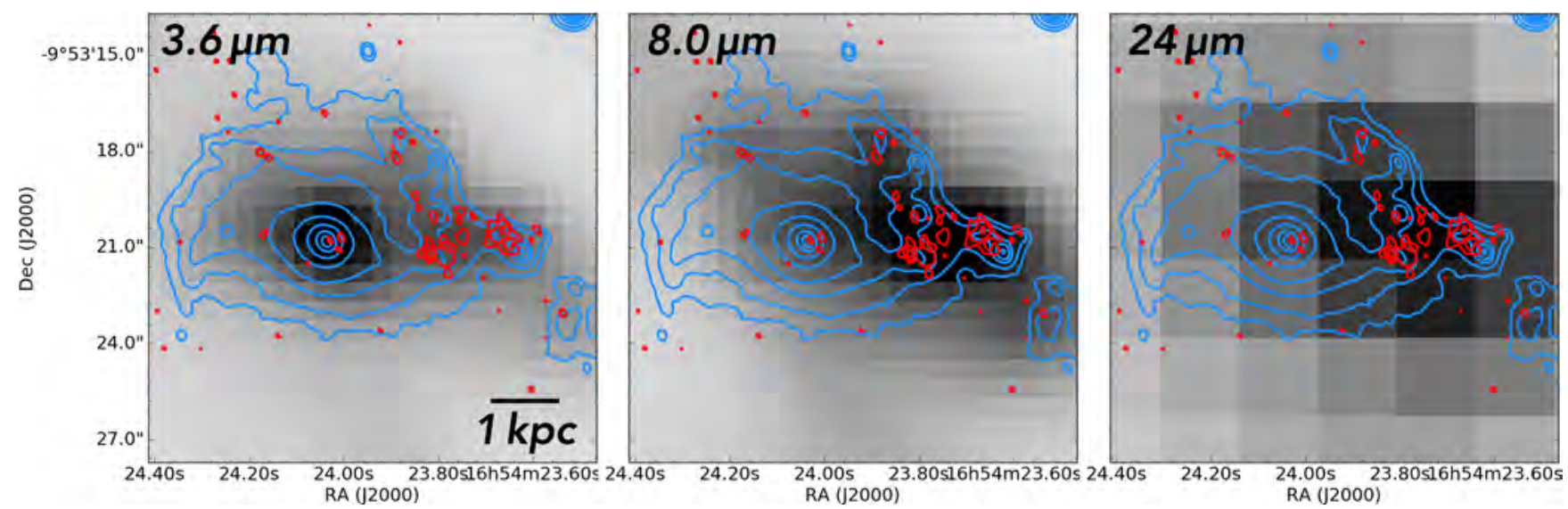

Figure 10. Near-IR and radio contours of IRAS 16516-0948 overplotted on Spitzer images. Left: IRAC $3.6 \mu \mathrm{m}$. Middle: IRAC $8.0 \mu \mathrm{m}$. Right: MIPS $24 \mu \mathrm{m}$. We have overplotted our near-IR $K$-band (blue) and radio $X$-band (red) contours. North is up, and east is left. Note how the peak IR emission clearly shifts to the west as wavelength increases.

\subsection{Radio spectral behaviour}

Fig. 11 shows the spectral index maps of the sources. The most prominent characteristic is the patchy spatial distribution of $\alpha$. This has been reported in the literature (e.g. in NGC 1596; see Lisenfeld et al. 2004), and the most likely explanation is that the patches show regions with different ages, therefore the thermal and non-thermal radio emission peaks are not spatially coincident, producing the observed distribution. The regions with the steepest spectrum are dominated by old radio emitters, while those with flatter spectrum correspond to regions with ongoing activity associated with recently exploded $\mathrm{SNe}$ and/or AGN activity.

Table 5 quotes the average spectral indices, masked below $10 \times \mathrm{rms}$ in the total intensity images to ensure robustness in the spectral index calculation. We note that the signal-to-noise ratio of the radio image of IRAS 16516-0948 is not good enough to create a reliable spectral index map, since only a few pixels are above the quoted $10 \times$ rms threshold.

We do not find a correlation between the spectral index maps and the emission ratios shown in Fig. 4. The median spectral index of our sample is -0.78 , consistent with the values derived for starforming galaxies at $L(\simeq 18 \mathrm{~cm})$ and $C$ bands $(\simeq 6 \mathrm{~cm})$ (Leroy et al. 2011; Basu et al. 2015), with a single power law describing the spectral behaviour. Thus, we find no significant flattening due to thermal contribution, whose emission has a characteristic spectral index of $\alpha \simeq-0.1$. We note, however, that this invariance in the spectral index does not hold for even lower frequencies, where free-free absorption mechanisms dominate (Clemens et al. 2010). This has already been shown for the case of Arp 220 with LOFAR observations (Varenius et al. 2016).

We find that, with the exception of NGC $3690 \mathrm{~W}$ and IC 883, those sources with a steeper spectral index $(\alpha<-0.8)$ show extended diffuse emission. Murphy et al. (2013) studied a sample of 36 compact SB finding the same trend. This can be explained with radio emission arising from radio continuum bridges and tidal tails, result of the interactions, which do not have a direct stellar origin; instead, its emission is due to relativistic electrons cooling down in those regions and producing the steep spectrum.

The steepest spectral index in our sample corresponds to IRAS 18293-3413, with $\alpha=-1.73$. While this source has the highest SFR from our SED modelling, this is an unexpectedly steep $\alpha$ value for an SB galaxy. Further observations are needed to obtain its radio spectrum at different bands, allowing us to disentangle the different components of the radio emission and, eventually, understanding the origin of this oddity.

\section{SUMMARY AND CONCLUSIONS}

We have characterized the star formation and AGN activity of a sample of 11 local LIRGs imaged with subarcsecond angular resolution at radio and near-infrared wavelengths. We have performed a complete SED model fit, using a combination of SB and AGN templates, aimed at isolating the spheroid, SB and AGN luminosities as well as to derive the star formation and $\mathrm{SN}$ rates, their age and their AGN contribution. We also compare SFR and AGN luminosities derived using different IR and radio tracers. Finally, we report new radio $(3.6 \mathrm{~cm})$ and near-IR $(2.2 \mu \mathrm{m})$ data of the central $\mathrm{kpc}$ region of our LIRG sample. The main results of our study are the following ones.

(i) From our SED modelling, we determine the luminosity contribution of each component, and find that all but one of the sources in our sample are SB-dominated, with a significant AGN contribution ( $\gtrsim 20$ percent) only in three of them. Only one source (NGC 6926) is AGN-dominated (64 per cent). We also derive SFRs (40-167 $\left.\mathrm{M}_{\odot} \mathrm{yr}^{-1}\right)$, SN rates $\left(0.4-2.0 \mathrm{SN} \mathrm{yr}^{-1}\right)$ and SB ages (23-29 Myr) that are consistent with their LIRG nature. The only exception is NGC 6926, the youngest SB (9 Myr) of our sample, in which massive stars did not have time to go off SNe yet.

(ii) We find an overall consistency among the different (IR and radio) star formation tracers, with no star formation tracer underestimating/overestimating systematically the SFR with respect to others. The only exception is the overestimated radio-derived SFR in NGC 6240, likely due to the strong dual AGN influence.

(iii) We also find an overall consistency between the common tracers of AGN contribution based on the mid-IR high-ionization line ratios $\left([\mathrm{NeV}] /[\mathrm{Ne} I I]\right.$ and $\left[\mathrm{O}_{\mathrm{IV}}\right] /[\mathrm{Ne} \mathrm{II}]$ versus $\left.\mathrm{PAH} \mathrm{EW}\right)$ and our predicted $\mathrm{SED}$ model fitted $\mathrm{L}_{\mathrm{AGN}}$. However, the AGN luminosities derived directly from [ $\mathrm{Ne} v]$ are not fully compatible with our SED-model derived luminosities, possibly because extreme stellar populations are contributing significantly to the [Ne v] emission.

(iv) From our subarcsecond imaging at radio $(8.4 \mathrm{GHz} \mathrm{VLA})$ and near-IR $(2.2 \mu \mathrm{m}$ NACO-VLT and ALTAIR-Gemini) observations, we find that, in general, there is a good spatial correlation of the radio and the near-IR emission, and that both tend to be more concentrated in the nuclear regions of the galaxies. The lack of a 

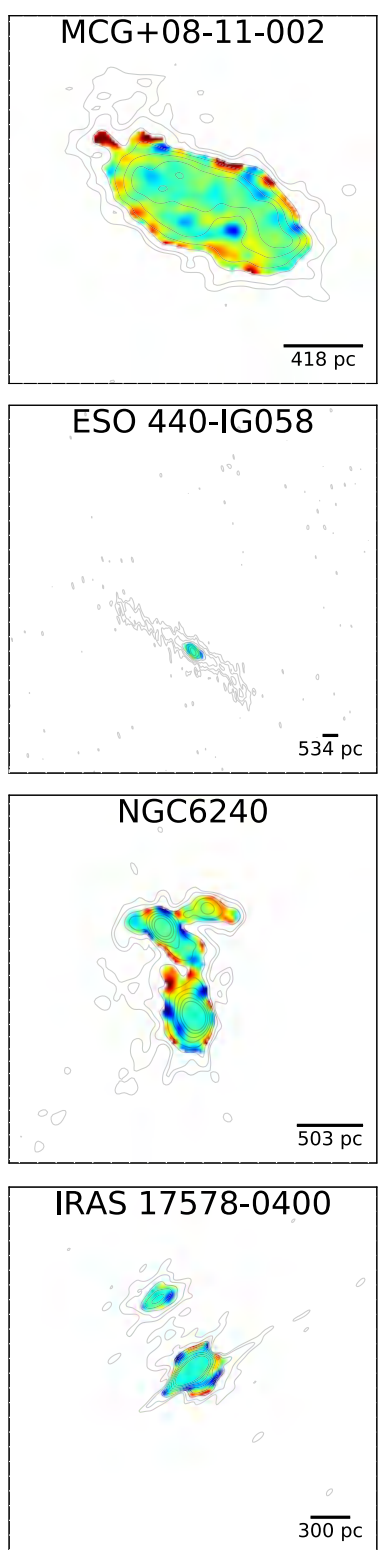
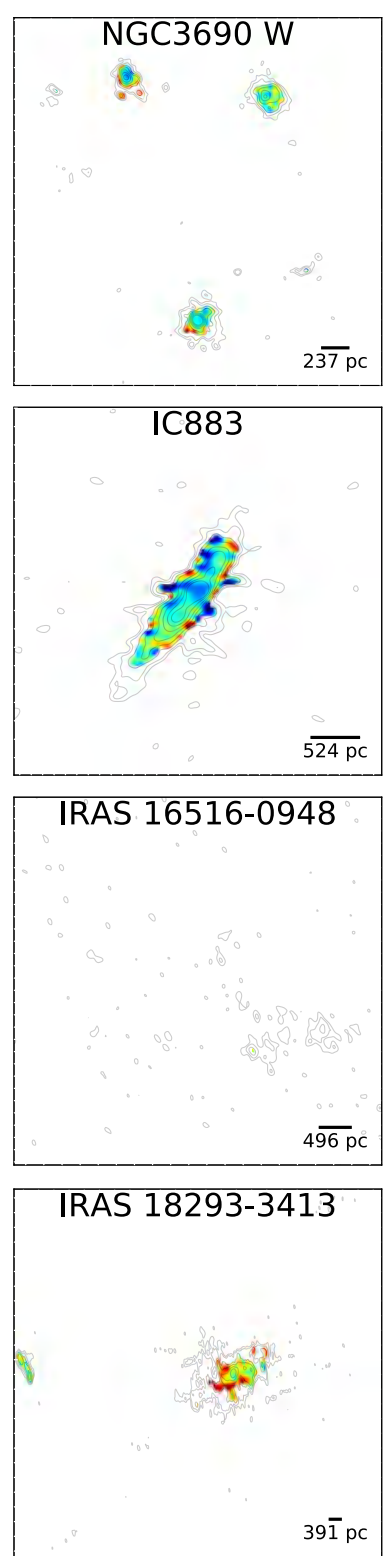
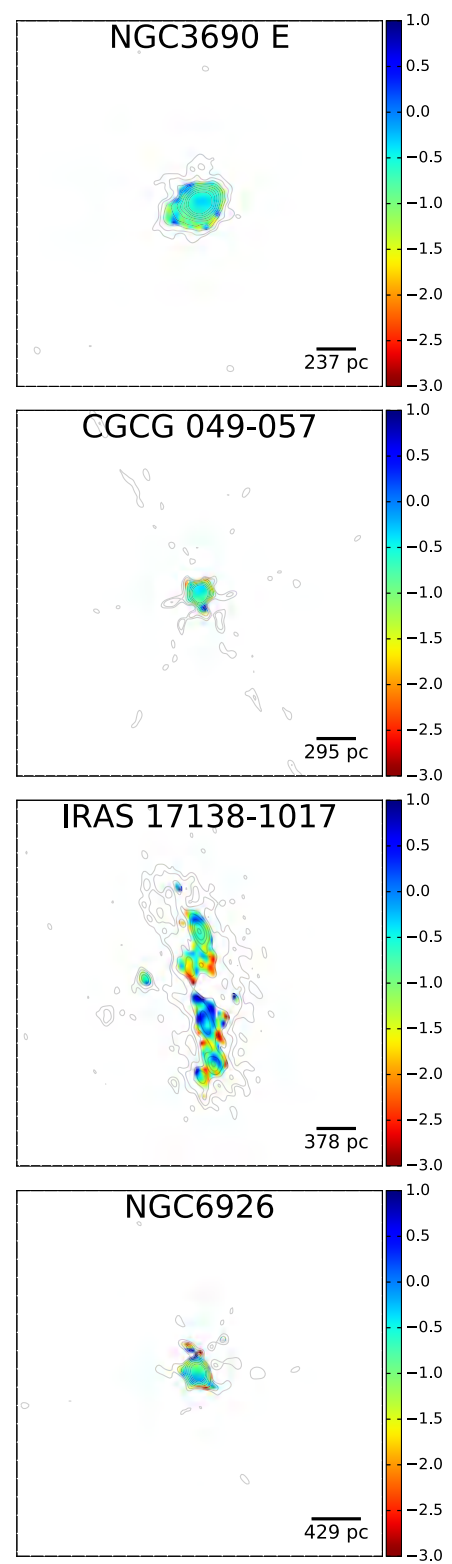

Figure 11. Spectral index maps of our sample. We have masked the maps in any point with a flux density below $10 \times$ rms to ensure the reliability of the spectral indices. Radio contours have been drawn as in Fig. 4. All the plots were made with the same colour scale. See the main text for details on how the maps were obtained.

radio counterpart for a few conspicuous near-IR features in some sources points to young SB with no SNe to produce synchrotron radiation. We note that we clearly identified one off-nuclear SB in the late-merger IRAS 16516-0948, which may have been likely triggered by the ongoing merging process.

(v) We have obtained spectral index maps, showing a patchy spatial distribution. We find an average spectral index of $\alpha \simeq-0.8\left(S_{v}\right.$ $\left.\propto v^{\alpha}\right)$ from our wide-band VLA observations. This is a typical value for synchrotron-powered LIRGs, and shows that the contribution of thermal emission to the total radio emission at $8.4 \mathrm{GHz}$ is very small.

\section{ACKNOWLEDGEMENTS}

RHI, MAPT and AA acknowledge support from the Spanish MINECO through grants AYA2012-38491-C02-02 and AYA2015-
63939-C2-1-P. PV acknowledges support from the National Research Foundation of South Africa. ZR thanks funding from the South African SKA. We thank the anonymous referee for his/her useful comments and feedback, which have improved the quality of this work. The authors also thank Cristina Romero-Cañizales and Lorena Hernández-García for the useful discussion. The National Radio Astronomy Observatory is a facility of the National Science Foundation operated under cooperative agreement by Associated Universities, Inc. Based in part on observations obtained at the Gemini Observatory (Program IDs: GN-2008A-Q-38, GN2008B-Q-32, GN-2009A-Q-12, GN-2009B-Q-23, GN-2010A-Q40, GN-2011A-Q-48, GN-2011B-Q-73, GN-2012A-Q-56 and GS2015A-Q-7), which is operated by the Association of Universities for Research in Astronomy, Inc., under a cooperative agreement with the NSF on behalf of the Gemini partnership: the National Science Foundation (United States), the National Research Council 
Table 5. Spectral indices.

\begin{tabular}{lcc}
\hline Name & Average $\alpha$ & Dispersion \\
\hline MCG+08-11-002 & -1.09 & 0.58 \\
NGC 3690W & -1.01 & 0.64 \\
NGC 3690E & -0.65 & 0.35 \\
ESO 440-IG058 & -0.60 & 0.36 \\
IC 883 & -0.68 & 0.90 \\
CGCG 049-057 & -0.78 & 0.54 \\
NGC 6240 & -0.99 & 0.76 \\
IRAS 16516-0948 & - & - \\
IRAS 17138-1017 & -0.85 & 0.80 \\
IRAS 17578-0400 & -0.64 & 0.70 \\
IRAS 18293-3413 & -1.73 & 0.70 \\
NGC 6926 & -0.76 & 1.00 \\
\hline
\end{tabular}

Note. The quoted dispersions correspond to the standard deviation of the individual (pixel) spectral indices, and are thus a measurement of the uniformity of the spectral index across the region.

(Canada), CONICYT (Chile), Ministerio de Ciencia, Tecnología e Innovación Productiva (Argentina) and Ministério da Ciência, Tecnologia e Inovação (Brazil). And based in part on observations made with the European Southern Observatory Telescopes, at the La Silla Paranal Observatory, Chile, under programmes 073.D-0406 (PI: Vaisanen), 087.D-0444 and 089.D-0847 (PI: Mattila). We acknowledge the use of the VizieR photometry tool, developed by Anne-Camille Simon and Thomas Boch.

\section{REFERENCES}

Abel N. P., Satyapal S., 2008, ApJ, 678, 686

Ahn C. P. et al., 2012, ApJS, 203, 21

Alonso-Herrero A., Rieke G. H., Rieke M. J., Scoville N. Z., 2000, ApJ, 532,845

Alonso-Herrero A., Engelbracht C. W., Rieke M. J., Rieke G. H., Quillen A. C., 2001, ApJ, 546, 952

Alonso-Herrero A. et al., 2014, MNRAS, 443, 2766

Armus L. et al., 2007, ApJ, 656, 148

Armus L. et al., 2009, PASP, 121, 559

Asmus D., Hönig S. F., Gandhi P., Smette A., Duschl W. J., 2014, MNRAS, 439, 1648

Baan W. A., Klöckner H.-R., 2006, A\&A, 449, 559

Baan W. A., Henkel C., Haschick A. D., 1987, ApJ, 320, 154

Ballo L., Braito V., Della Ceca R., Maraschi L., Tavecchio F., Dadina M., 2004, ApJ, 600, 634

Bassani L., Dadina M., Maiolino R., Salvati M., Risaliti G., Della Ceca R., Matt G., Zamorani G., 1999, ApJS, 121, 473

Basu A., Roy S., 2013, MNRAS, 433, 1675

Basu A., Beck R., Schmidt P., Roy S., 2015, MNRAS, 449, 3879

Beswick R. J., Pedlar A., Mundell C. G., Gallimore J. F., 2001, MNRAS, 325, 151

Bondi M., Pérez-Torres M. A., Herrero-Illana R., Alberdi A., 2012, A\&A, 539, A134

Bonzini M. et al., 2015, MNRAS, 453, 1079

Bottinelli L., Gouguenheim L., Le Squeren A. M., Martin J. M., Dennefeld M., Paturel G., 1986, IAU Circ., 4231, 2

Brandl B. R. et al., 2006, ApJ, 653, 1129

Bruzual G., Charlot S., 1993, ApJ, 405, 538

Bruzual G., Charlot S., 2003, MNRAS, 344, 1000

Calistro Rivera G., Lusso E., Hennawi J. F., Hogg D. W., 2016, ApJ, 833, 98

Cleary K., Lawrence C. R., Marshall J. A., Hao L., Meier D., 2007, ApJ, 660,117

Clemens M. S., Scaife A., Vega O., Bressan A., 2010, MNRAS, 405, 887

Colbert E. J. M., Wilson A. S., Bland-Hawthorn J., 1994, ApJ, 436, 89

Condon J. J., 1992, ARA\&A, 30, 575
Condon J. J., Broderick J. J., 1991, AJ, 102, 1663

Condon J. J., Yin Q. F., 1990, ApJ, 357, 97

Condon J. J., Cotton W. D., Greisen E. W., Yin Q. F., Perley R. A., Taylor G. B., Broderick J. J., 1998, AJ, 115, 1693

Corbett E. A. et al., 2002, ApJ, 564, 650

Corbett E. A. et al., 2003, ApJ, 583, 670

Corwin H. G., 2004, VizieR Online Data Catalog, 7239, 0

Costagliola F., Herrero-Illana R., Lohfink A., Pérez-Torres M., Aalto S., Muller S., Alberdi A., 2016, A\&A, 594, A114

Dametto N. Z., Riffel R., Pastoriza M. G., Rodríguez-Ardila A., HernandezJimenez J. A., Carvalho E. A., 2014, MNRAS, 443, 1754

Davies R. L., Medling A. M. U V., Max C. E., Sanders D., Kewley L. J., 2016, MNRAS, 458, 158

de Jong T., Klein U., Wielebinski R., Wunderlich E., 1985, A\&A, 147, L6

Del Moro A. et al., 2013, A\&A, 549, A59

Della Ceca R. et al., 2002, ApJ, 581, L9

Depoy D. L., Wynn-Williams C. G., Hill G. J., Becklin E. E., 1988, AJ, 95, 398

Diamond-Stanic A. M., Rieke G. H., Rigby J. R., 2009, ApJ, 698, 623

Díaz-Santos T., Alonso-Herrero A., Colina L., Ryder S. D., Knapen J. H., 2007, ApJ, 661, 149

Díaz-Santos T. et al., 2011, ApJ, 741, 32

Dixon T. G., Joseph R. D., 2011, ApJ, 740, 99

Donley J. L., Rieke G. H., Rigby J. R., Pérez-González P. G., 2005, ApJ, 634,169

Efstathiou A., 2006, MNRAS, 371, L70

Efstathiou A., Rowan-Robinson M., 1995, MNRAS, 273, 649

Efstathiou A., Rowan-Robinson M., 2003, MNRAS, 343, 322

Efstathiou A., Siebenmorgen R., 2009, A\&A, 502, 541

Efstathiou A., Rowan-Robinson M., Siebenmorgen R., 2000, MNRAS, 313 , 734

Efstathiou A., Christopher N., Verma A., Siebenmorgen R., 2013, MNRAS, 436, 1873

Engelbracht C. W., Rieke M. J., Rieke G. H., Latter W. B., 1996, ApJ, 467, 227

Farrah D. et al., 2007, ApJ, 667, 149

Gallimore J. F., Beswick R., 2004, AJ, 127, 239

Gehrz R. D., Sramek R. A., Weedman D. W., 1983, ApJ, 267, 551

Genzel R. et al., 1998, ApJ, 498, 579

Greenhill L. J., Kondratko P. T., Lovell J. E. J., Kuiper T. B. H., Moran J. M., Jauncey D. L., Baines G. P., 2003, ApJ, 582, L11

Haan S. et al., 2011, AJ, 141, 100

Heckman T. M., Lehnert M. D., Armus L., 1993, in Shull J. M., Thronson H. A., eds, Astrophysics and Space Science Library, Vol. 188, The Environment and Evolution of Galaxies. Kluwer, Dordrecht, p. 455

Heckman T. M., Ptak A., Hornschemeier A., Kauffmann G., 2005, ApJ, 634, 161

Helou G., Soifer B. T., Rowan-Robinson M., 1985, ApJ, 298, L7

Herrero-Illana R., Pérez-Torres M. Á., Alberdi A., 2012a, A\&A, 540, L5

Herrero-Illana R., Romero-Canizales C., Perez-Torres M. A., Alberdi A., Kankare E., Mattila S., Ryder S. D., 2012b, Astron. Telegram, 4432, 1

Herrero-Illana R. et al., 2014, ApJ, 786, 156

Inami H. et al., 2010, AJ, 140, 63

Inami H. et al., 2013, ApJ, 777, 156

Ivison R. J. et al., 2010, A\&A, 518, L31

Johnson S. P., Wilson G. W., Tang Y., Scott K. S., 2013, MNRAS, 436, 2535

Kankare E. et al., 2008a, ApJ, 689, L97

Kankare E. et al., 2008b, Cent. Bur. Electron. Telegrams, 1569

Kankare E. et al., 2012, ApJ, 744, L19

Kankare E. et al., 2014, MNRAS, 440, 1052

Keel W. C., Wu W., 1995, AJ, 110, 129

Kennicutt R. C., Jr, 1998, ARA\&A, 36, 189

Kewley L. J., Dopita M. A., Sutherland R. S., Heisler C. A., Trevena J., 2001, ApJ, 556, 121

Kool E. et al., 2017, MNRAS, preprint (arXiv:e-prints)

Komossa S., Burwitz V., Hasinger G., Predehl P., Kaastra J. S., Ikebe Y., 2003, ApJ, 582, L15

Koss M., Mushotzky R., Baumgartner W., Veilleux S., Tueller J., Markwardt C., Casey C. M., 2013, ApJ, 765, L26 
Lacki B. C., Thompson T. A., 2010, ApJ, 717, 196

Lacki B. C., Thompson T. A., Quataert E., 2010, ApJ, 717, 1

Larson K. L. et al., 2016, ApJ, 825, 128

Lehmer B. D., Alexander D. M., Bauer F. E., Brandt W. N., Goulding A. D., Jenkins L. P., Ptak A., Roberts T. P., 2010, ApJ, 724, 559

Leitherer C., Heckman T. M., 1995, ApJS, 96, 9

Leroy A. K. et al., 2011, ApJ, 739, L25

Li W. D., 2002, IAU Circ., 7864, 2

Lisenfeld U., Voelk H. J., Xu C., 1996, A\&A, 314, 745

Lisenfeld U., Wilding T. W., Pooley G. G., Alexander P., 2004, MNRAS, 349,1335

Lu N. et al., 2014, ApJ, 787, L23

McMullin J. P., Waters B., Schiebel D., Young W., Golap K., 2007, in Shaw R. A., Hill F., Bell D. J., eds, ASP Conf. Ser. Vol. 376, Astronomical Data Analysis Software and Systems XVI. Astron. Soc. Pac., San Francisco, p. 127

Maraston C., 1998, MNRAS, 300, 872

Masini A. et al., 2016, A\&A, 589, A59

Matheson T., Jha S., Challis P., Kirshner R., Calkins M., 2002, IAU Circ., 7872,2

Mattila S., Meikle W. P. S., 2001, MNRAS, 324, 325

Mattila S. et al., 2007a, ApJ, 659, L9

Mattila S. et al., 2007b, Cent. Bur. Electron. Telegrams, 858, 1

Mattila S., Kankare E., Datson J., Pastorello A., 2010, Cent. Bur. Electron. Telegrams, 2149, 1

Mattila S. et al., 2012, ApJ, 756, 111

Mauch T., Sadler E. M., 2007, MNRAS, 375, 931

Miluzio M. et al., 2013, A\&A, 554, A127

Modica F. et al., 2012, AJ, 143, 16

Monreal-Ibero A., Arribas S., Colina L., Rodríguez-Zaurín J., AlonsoHerrero A., García-Marín M., 2010, A\&A, 517, A28

Morić I., Smolčić V., Kimball A., Riechers D. A., Ivezić Ž., Scoville N., 2010, ApJ, 724, 779

Mullaney J. R., Alexander D. M., Goulding A. D., Hickox R. C., 2011, MNRAS, 414, 1082

Murphy E. J. et al., 2011, ApJ, 737, 67

Murphy E. J., Stierwalt S., Armus L., Condon J. J., Evans A. S., 2013, ApJ, 768,2

Nakai N., Sato N., Yamauchi A., 2002, PASJ, 54, L27

Neff S. G., Ulvestad J. S., Teng S. H., 2004, ApJ, 611, 186

Netzer H. et al., 2007, ApJ, 666, 806

Oliva E., Origlia L., Kotilainen J. K., Moorwood A. F. M., 1995, A\&A, 301, 55

Oliva E., Moorwood A. F. M., Drapatz S., Lutz D., Sturm E., 1999, A\&A, 343, 943

Padovani P., Miller N., Kellermann K. I., Mainieri V., Rosati P., Tozzi P., 2011, ApJ, 740, 20

Pannella M. et al., 2015, ApJ, 807, 14

Parra R., Conway J. E., Diamond P. J., Thrall H., Lonsdale C. J., Lonsdale C. J., Smith H. E., 2007, ApJ, 659, 314

Pereira-Santaella M., Diamond-Stanic A. M., Alonso-Herrero A., Rieke G. H., 2010, ApJ, 725, 2270

Pérez-Torres M. A. et al., 2007, ApJ, 671, L21

Pérez-Torres M. A., Romero-Cañizales C., Alberdi A., Polatidis A., 2009, A\&A, 507, L17

Pérez-Torres M. A., Alberdi A., Romero-Cañizales C., Bondi M., 2010, A\&A, 519, L5

Petric A. O. et al., 2011, ApJ, 730, 28

Portegies Zwart S. F., McMillan S. L. W., Gieles M., 2010, ARA\&A, 48, 431

Randriamanakoto Z., Väisänen P., Ryder S., Kankare E., Kotilainen J., Mattila S., 2013a, MNRAS, 431, 554

Randriamanakoto Z., Escala A., Väisänen P., Kankare E., Kotilainen J., Mattila S., Ryder S., 2013b, ApJ, 775, L38

Rau U., Cornwell T. J., 2011, A\&A, 532, A71

Rich J. A., Kewley L. J., Dopita M. A., 2015, ApJS, 221, 28

Rieke G. H., Loken K., Rieke M. J., Tamblyn P., 1993, ApJ, 412, 99

Risaliti G., Gilli R., Maiolino R., Salvati M., 2000, A\&A, 357, 13
Risaliti G. et al., 2006, ApJ, 637, L17

Rodríguez-Zaurín J., Arribas S., Monreal-Ibero A., Colina L., AlonsoHerrero A., Alfonso-Garzón J., 2011, A\&A, 527, A60

Romero-Cañizales C., Mattila S., Alberdi A., Pérez-Torres M. A., Kankare E., Ryder S. D., 2011, MNRAS, 415, 2688

Romero-Cañizales C., Pérez-Torres M. Á., Alberdi A., 2012a, MNRAS, 422,510

Romero-Cañizales C. et al., 2012b, A\&A, 543, A72

Romero-Cañizales C. et al., 2014, MNRAS, 440, 1067

Romero-Cañizales C. et al., 2017, MNRAS, 467, 2504

Roy A. L., Norris R. P., 1997, MNRAS, 289, 824

Ryder S., Mattila S., Kankare E., Perez-Torres M., 2010, Cent. Bur. Electron. Telegrams, 2189, 1

Sales D. A., Pastoriza M. G., Riffel R., 2010, ApJ, 725, 605

Sanders D. B., Mazzarella J. M., Kim D.-C., Surace J. A., Soifer B. T., 2003 , AJ, 126, 1607

Sato N., Yamauchi A., Ishihara Y., Sorai K., Kuno N., Nakai N., Balasubramanyam R., Hall P., 2005, PASJ, 57, 587

Satyapal S., Vega D., Heckman T., O'Halloran B., Dudik R., 2007, ApJ, 663, L9

Scoville N. Z. et al., 2000, AJ, 119, 991

Shao L. et al., 2010, A\&A, 518, L26

Shipley H. V., Papovich C., Rieke G. H., Brown M. J. I., Moustakas J., 2016, ApJ, 818, 60

Smith D. A., Herter T., Haynes M. P., Beichman C. A., Gautier T. N., III, 1995, ApJ, 439, 623

Stierwalt S. et al., 2013, ApJS, 206, 1

Stierwalt S. et al., 2014, ApJ, 790, 124

Sturm E., Lutz D., Tran D., Feuchtgruber H., Genzel R., Kunze D., Moorwood A. F. M., Thornley M. D., 2000, A\&A, 358, 481

Tabatabaei F. S., Beck R., Krügel E., Krause M., Berkhuijsen E. M., Gordon K. D., Menten K. M., 2007, A\&A, 475, 133

Tadhunter C. et al., 2007, ApJ, 661, L13

Terashima Y., Hirata Y., Awaki H., Oyabu S., Gandhi P., Toba Y., Matsuhara H., 2015, ApJ, 814, 11

Treister E., Urry C. M., Virani S., 2009, ApJ, 696, 110

Ulvestad J. S., 2009, AJ, 138, 1529

Väisänen P. et al., 2008a, MNRAS, 384, 886

Väisänen P., Ryder S., Mattila S., Kotilainen J., 2008b, ApJ, 689 , L37

Väisänen P., Rajpaul V., Zijlstra A. A., Reunanen J., Kotilainen J., 2012, MNRAS, 420, 2209

Väisänen P., Reunanen J., Kotilainen J., Mattila S., Johansson P. H., Ramphul R., Romero-Cañizales C., 2017, MNRAS, preprint (arXiv:1701.00890) van der Kruit P. C., 1973, A\&A, 29, 263

Vardoulaki E. et al., 2015, A\&A, 574, A4

Varenius E. et al., 2016, A\&A, 593, A86

Vega O., Clemens M. S., Bressan A., Granato G. L., Silva L., Panuzzo P., 2008, A\&A, 484, 631

Veilleux S., Kim D.-C., Sanders D. B., Mazzarella J. M., Soifer B. T., 1995, ApJS, 98, 171

Voelk H. J., 1989, A\&A, 218, 67

Voit G. M., 1992, MNRAS, 258, 841

Weedman D. W. et al., 2005, ApJ, 633, 706

Wu Y., Charmandaris V., Huang J., Spinoglio L., Tommasin S., 2009, ApJ, 701,658

Yuan T.-T., Kewley L. J., Sanders D. B., 2010, ApJ, 709, 884

Yun M. S., Reddy N. A., Condon J. J., 2001, ApJ, 554, 803

Zezas A., Ward M. J., Murray S. S., 2003, ApJ, 594, L31

\section{APPENDIX: NON-SMOOTHED NEAR-IR IMAGES WITH RADIO CONTOURS}

We show in Fig. A1, the original non-smoothed near-IR images with the overlaid contours corresponding to the radio emission. 

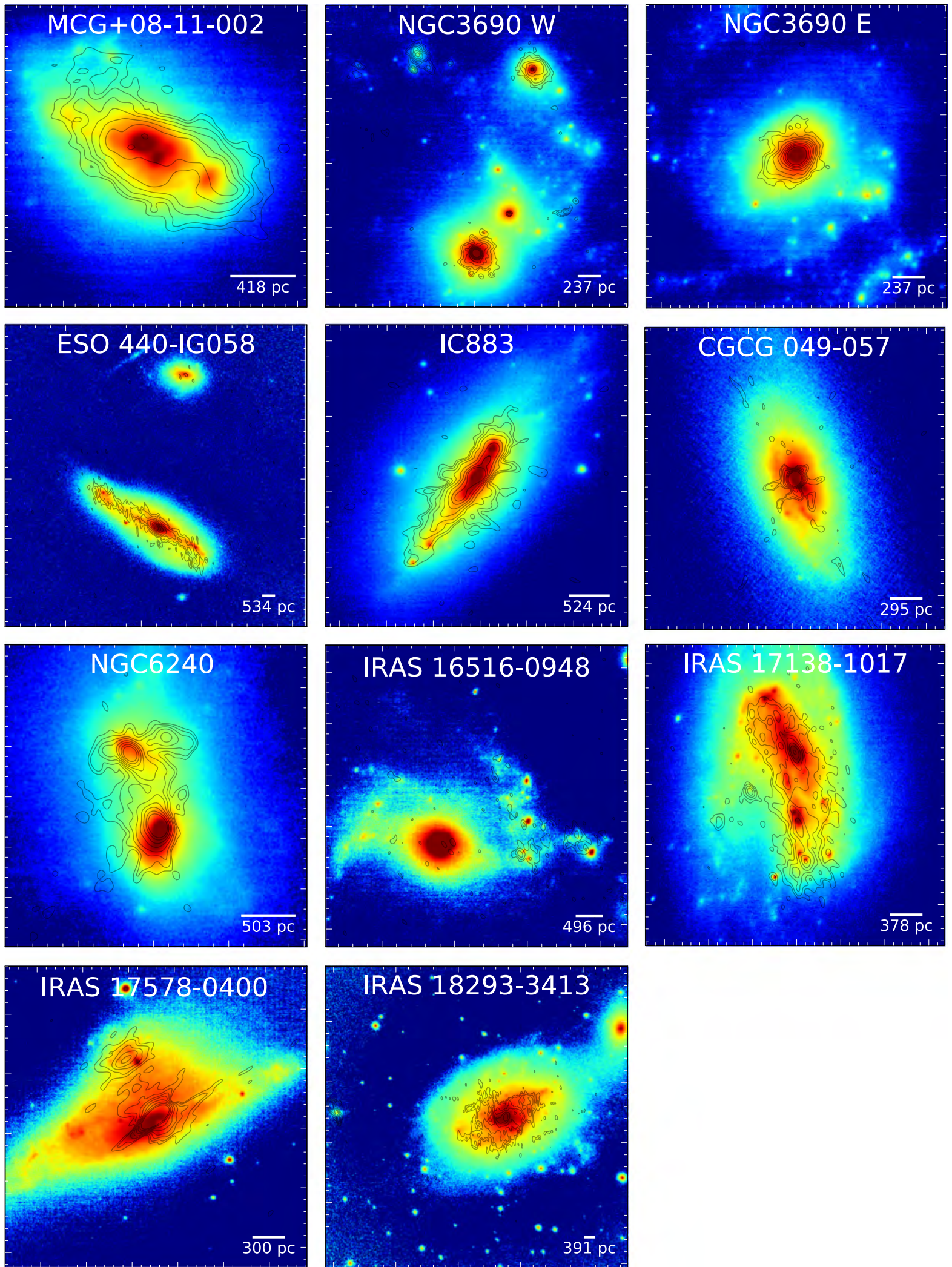

Figure A1. $2.2 \mu \mathrm{m}$ non-smoothed near-IR images with overlaid $3.6 \mathrm{~cm}$ radio contours. Contours are drawn every $3(\sqrt{3})^{n} \times \mathrm{rms}$ level, with $n=0,1,2,3$, 